\title{
PROTOSTELLAR OUTFLOWS IN L1340
}

\author{
Josh Walawender ${ }^{1}$, Grace Wolf-Chase ${ }^{2}$, Michael Smutko ${ }^{3}$, JoAnn OLinger-Luscusk ${ }^{4}$, And Gerald Moriarty-Schieven ${ }^{5}$ \\ ${ }^{1}$ W. M. Keck Observatory, 65-1120 Mamalahoa Hwy, Kamuela, HI 96743, USA; jmwalawender@keck.hawaii.edu \\ ${ }^{2}$ Astronomy Department, Adler Planetarium, 1300 South Lake Shore Drive, Chicago, IL 60605, USA \\ ${ }^{3}$ Center for Interdisciplinary Exploration and Research in Astrophysics (CIERA) \& Department of Physics \& Astronomy, Northwestern University, 2145 Sheridan \\ Road, Evanston, IL 60208, USA \\ ${ }^{4}$ California Institute of Technology, 1200 E California Blvd, Pasadena, CA 91125, USA \\ ${ }^{5}$ National Research Council-Herzberg Astronomy \& Astrophysics, 5017 West Saanich Road, Victoria, BC, V9E 2E7, Canada \\ Received 2016 April 21; revised 2016 September 23; accepted 2016 September 28; published 2016 November 30
}

\begin{abstract}
We have searched the L1340 A, B, and C clouds for shocks from protostellar outflows using the $\mathrm{H}_{2} 2.122 \mu$ m nearinfrared line as a shock tracer. Substantial outflow activity has been found in each of the three regions of the cloud (L1340 A, L1340 B, and L1340 C). We find 42 distinct shock complexes (16 in L1340 A, 11 in L1340 B, and 15 in L1340 C). We were able to link 17 of those shock complexes into 12 distinct outflows and identify candidate source stars for each. We examine the properties $\left(A_{\mathrm{V}}, T_{\mathrm{bol}}\right.$, and $\left.L_{\mathrm{bol}}\right)$ of the source protostars and compare them to the properties of the general population of Class $0 / \mathrm{I}$ and flat spectral energy distribution protostars and find that there is an indication, albeit at low statistical significance, that the outflow-driving protostars are drawn from a population with lower $A_{\mathrm{V}}$, higher $L_{\mathrm{bol}}$, and lower $T_{\mathrm{bol}}$ than the general population of protostars.
\end{abstract}

Key words: Herbig-Haro objects - ISM: jets and outflows - stars: formation - stars: jets - stars: pre-main sequence - stars: protostars

\section{INTRODUCTION}

Young stars interact with their parent molecular cloud through the action of protostellar outflows. These magnetohydrodynamically driven outflows are launched at speeds of order $100 \mathrm{~km} \mathrm{~s}^{-1}$ as a by-product of the accretion process. This high-velocity gas shocks against the surrounding material and is detected via shock tracers in the optical (such as $\mathrm{H} \alpha$ and [S II], in which case the shocks are called Herbig-Haro or $\mathrm{HH}$ objects) or in the infrared (typically via $\mathrm{H}_{2}$ or [Fe II] emission). These outflows affect the parent cloud's dynamics by opening cavities (e.g., Quillen et al. 2005) or by driving turbulence (e.g., Miesch \& Bally 1994; Bally et al. 1999; Arce \& Goodman 2002; Walawender et al. 2005).

We have surveyed the L1340 A, B, and C clouds in the nearinfrared (near-IR) to look for shocks from protostellar outflows as revealed by their $\mathrm{H}_{2} 2.12 \mu \mathrm{m} \mathrm{S}(1-0)$ emission. We found 42 shock complexes (MHO 2925 to 2966 in the MHO catalog hosted by the University of $\mathrm{Kent}^{6}$ and initially published in Davis et al. 2010).

LDN 1340 (L1340 hereafter) is an intermediate-mass (mid-B and lower luminosity) star-forming region in Cassiopeia. It was number 1340 of 1802 in Lynds' (1962) catalog of dark nebulae. Dorschner \& Gürtler (1963) cataloged a reflection nebula (DG 9) in the dark cloud. Later, Cohen (1980) examined Palomar Sky Survey images and found three "red nebulous objects" (RNO 7, 8, and 9) in the area.

The first detailed study of the area was carried out by Kun et al. (1994), who mapped the region in ${ }^{13} \mathrm{CO}$ and $\mathrm{C}^{18} \mathrm{O}$ and found a molecular mass of about $1300 M_{\odot}$. Based on their molecular maps, Kun et al. (1994) divided the L1340 cloud into three subregions: $\mathrm{L} 1340 \mathrm{~A}, \mathrm{~B}$, and $\mathrm{C}$, corresponding roughly to the areas surrounding RNO 7, 8, and 9, respectively.

Yonekura et al. (1997) included L1340 in their ${ }^{13} \mathrm{CO}$ survey of molecular clouds toward Cepheus and Cassiopeia and found

\footnotetext{
6 http://astro.kent.ac.uk/ df/MHCat/
}

a mass of $1200 M_{\odot}$. Kun et al. (2003) observed L1340 in $\mathrm{NH}_{3}$ and found 10 dense cores with a total mass of about $80 M_{\odot}$. Juvela et al. (2012) included L1340 in their Herschel SPIRE observations and described the morphology of the cloud as filamentary rather than cometary.

Kun et al. (2011) performed photometric and spectroscopic monitoring of the HA11 star (using the nomenclature of Kun et al. 1994) and found it to be an eruptive young star, though not easily falling into a classification as either an FUor or EXor type. Kun et al. (2014) discovered three additional candidate eruptive young stars in L1340: IRAS 02224+7227 (discussed in Section 3.1.1), 2MASS 02263797+7304575, and 2MASS $02325605+7246055$ (discussed in Section 3.3.5).

Two previous optical/near-IR surveys have been done in this region: Kumar et al. (2002) and Magakian et al. (2003). Kumar et al. (2002) found three HH objects in L1340 A. Magakian et al. (2003) found two additional HH objects in L1340 A and also cataloged $14 \mathrm{H} \alpha$ emission line stars (many overlapping with the list compiled by Kun et al. 1994).

Kun et al. (2016a) used optical spectroscopy and infrared and optical photometry to examine the protostellar population in L1340 and mapped the extinction in the region based on SDSS photometry. In addition, they use spectral classification of two zero-age main-sequence (ZAMS) stars associated with the cloud to estimate a distance of $825_{-80}^{+110} \mathrm{pc}$.

Using infrared photometry, Kun et al. (2016b) identified and classified 170 Class II, 27 flat spectral energy distribution (SED), and 45 Class 0/I protostars in L1340. They estimated the extinction to each using the results of Kun et al. (2016a) and derived bolometric temperatures and luminosities based on the SED of each protostar.

In the following sections, we describe our observations (Section 2) and give detailed descriptions of the outflows in each of the A (Section 3.1), B (Section 3.2), and C (Section 3.3) clouds. In Section 4 we compare L1340 with our prior observations of Barnard 1 (Section 4.1) and compare the properties of the outflows we have identified in L1340 with the 


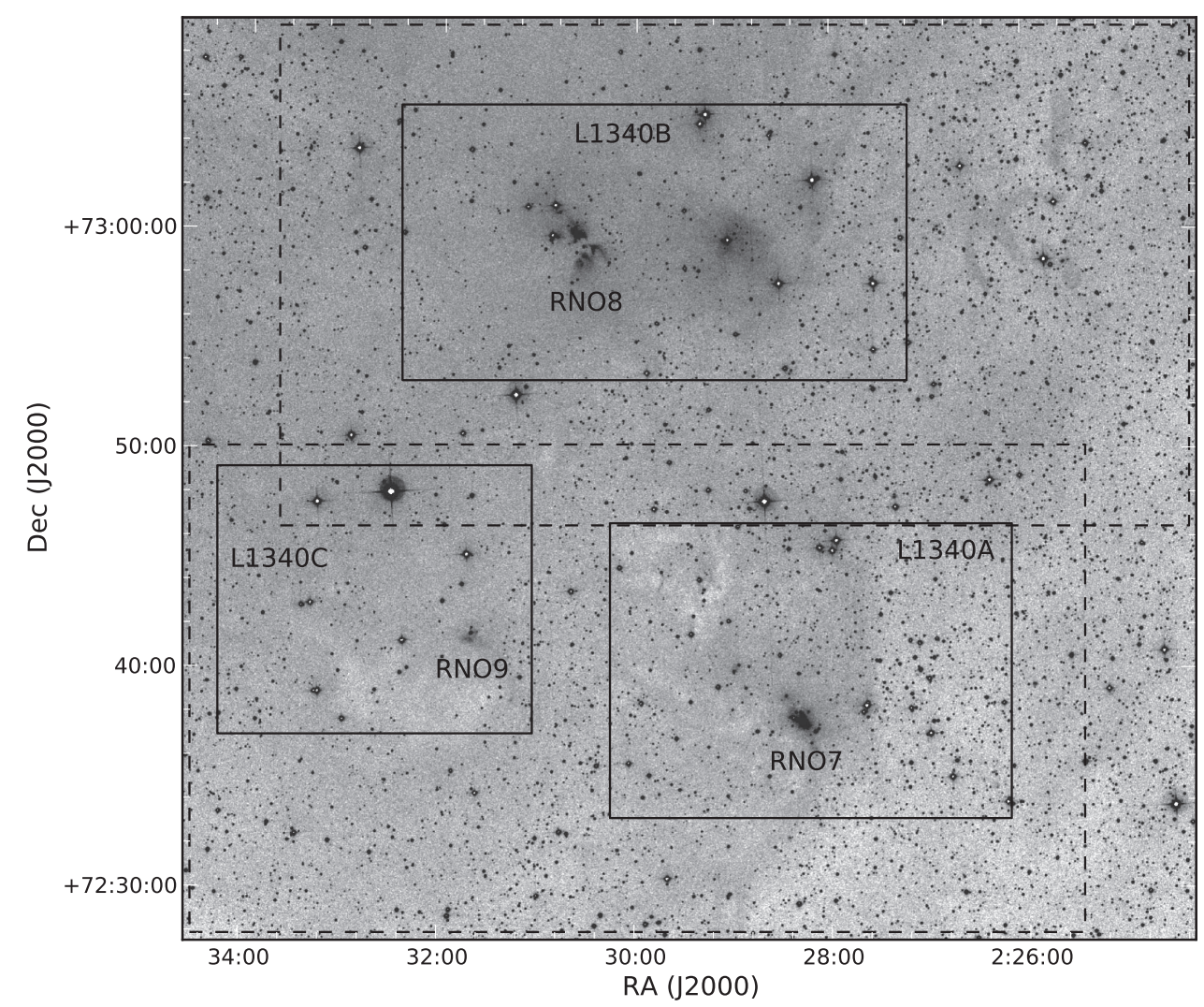

Figure 1. POSS R image of the L1340 cloud region. The three RNO objects of Cohen (1980) are labeled, and the three regions of the cloud (L1340 A, L1340 B, and L1340 C) are also marked. Boxes with dashed outlines indicate the rough coverage of our WIRCam $\mathrm{H}_{2}$ images, while boxes with solid outlines indicate the positions of Figures 2, 7, and 12 discussed later in the text.

properties of the protostars that drive them (Section 4.2). Our results are summarized in Section 5.

\section{OBSERVATIONS}

Data for this paper were taken during the 2006B and 2007B semesters with the Canada-France-Hawaii Telescope (CFHT) on Mauna Kea on the island of Hawai'i. The instrument used was the Wide-field InfraRed Camera (WIRCam; Puget et al. 2004), which consists of a mosaic of four HAWAII2RG detectors, each containing $2048 \times 2048$ pixels, with a sampling of 0.3 arcsecond per pixel on the sky. The data were pipeline processed by 'I'iwi, the standard WIRCam pipeline at Traitement Élémentaire, Réduction et Analyse des PIXels (TERAPIX), which is located at Institut d'Astrophysique de Paris (IAP).

Our 2006B data cover the northern half of the cloud (L1340 B) in $\mathrm{H}_{2}, \mathrm{H}$, and $\mathrm{K}_{S}$, while our 2007B data cover the southern half of the cloud (L1340 A and L1340 C) in $\mathrm{H}_{2}$ and all of the cloud in $\mathrm{J}, \mathrm{H}$, and $\mathrm{K}_{S}$ (see Figure 1). The total integration time in $\mathrm{H}_{2}$ is 54 minutes at each position. The integration times for the broadband filters vary depending on the position because some frames were rejected from the stacks due to poor image quality, but the minimum integration times were 3.5 minutes in J, 3.3 minutes in $\mathrm{H}$, and 3.67 minutes in $\mathrm{K}_{S}$. The seeing values in the final stacked images (as reported by the pipeline software) range from 0 ". 75 to 1 ". 55 FWHM. The total area covered is about 1700 square arcmin (0.47 square degrees) in each filter.

WIRCam images in all filters were registered to one another using their world coordinate system, and the $\mathrm{H}_{2}$ images of the two halves of the cloud were stitched together using the MSCRED package in the Image Reduction and Analysis Facility $^{7}$ (IRAF). In addition, IRAF was used to generate difference images $\left(\mathrm{H}_{2}-\mathrm{K}_{S}\right)$ after application of a Gaussian blur (to more closely match point-spread function (PSF) sizes) and a scaling factor (which was estimated from the flux of stars and reflection nebulae common to both images).

In addition, $\mathrm{H}_{2}$ and $\mathrm{K}_{S}$ images of a subregion of the $\mathrm{L} 1340 \mathrm{~B}$ cloud were obtained at the ARC $3.5 \mathrm{~m}$ telescope at Apache Point Observatory in New Mexico using the Near-Infrared Camera and Fabry-Perot Spectrometer (NICFPS; Hearty et al. 2004) instrument on the nights of 2005 September 19 and 2006 January 9-13. NICFPS uses a $1024 \times 1024$ pixel Hawaii-1RG detector, which has a pixel scale of 0.273 arcsec per pixel on the sky. The NICFPS images were processed and then registered with the WIRCam images using IRAF. The NICFPS data cover about 221 square arcmin $(0.06$ square degrees) in both $\mathrm{H}_{2}$ and $\mathrm{K}_{S}$.

\section{RESULTS}

We find 42 distinct shock complexes (16 in L1340 A, 11 in L1340 B, and 15 in L1340 C). Each shock complex is given an MHO designation (Davis et al. 2010), which is listed in Table 1, and each is discussed below. We group the discussions by region: L1340 A is covered in Section 3.1, L1340 B in Section 3.2, and L1340 C in Section 3.3.

\footnotetext{
IRAF is distributed by the National Optical Astronomy Observatories, which are operated by the Association of Universities for Research in Astronomy, Inc., under cooperative agreement with the National Science Foundation.
} 
Table 1

MHOs in L1340

\begin{tabular}{|c|c|c|c|}
\hline MHO Designation & R.A. (J2000) & Decl. (J2000) & Region \\
\hline MHO 2925A & $2: 26: 21.0$ & $72: 34: 37.1$ & L1340A \\
\hline 2925B & $2: 26: 19.9$ & $72: 35: 08.3$ & L1340A \\
\hline $2925 \mathrm{C}$ & $2: 26: 27.8$ & $72: 34: 57.3$ & L1340A \\
\hline 2925D & $2: 26: 31.2$ & $72: 35: 41.8$ & L1340A \\
\hline $2925 \mathrm{E}$ & $2: 26: 36.1$ & $72: 35: 12.7$ & L1340A \\
\hline $2925 \mathrm{~F}$ & $2: 26: 39.0$ & $72: 35: 26.0$ & L1340A \\
\hline $2925 \mathrm{G}$ & $2: 26: 39.5$ & $72: 36: 18.2$ & L1340A \\
\hline $2925 \mathrm{H}$ & $2: 26: 45.5$ & $72: 35: 41.9$ & L1340A \\
\hline MHO 2926A & $2: 27: 33.5$ & $72: 34: 08.4$ & L1340A \\
\hline 2926B & $2: 27: 29.6$ & $72: 34: 15.1$ & L1340A \\
\hline MHO 2927 & $2: 27: 39.0$ & $72: 35: 28.3$ & L1340A \\
\hline MHO 2928A & $2: 27: 56.4$ & $72: 35: 58.7$ & L1340A \\
\hline 2928B & $2: 27: 59.1$ & $72: 35: 53.9$ & L1340A \\
\hline $2928 \mathrm{C}$ & $2: 27: 59.8$ & $72: 35: 57.9$ & L1340A \\
\hline 2928D & $2: 28: 00.3$ & $72: 35: 55.7$ & L1340A \\
\hline $2928 \mathrm{E}$ & $2: 28: 06.2$ & $72: 35: 29.2$ & L1340A \\
\hline $2928 \mathrm{~F}$ & $2: 28: 15.2$ & $72: 35: 09.3$ & L1340A \\
\hline $2928 \mathrm{G}$ & $2: 28: 22.6$ & $72: 34: 55.6$ & L1340A \\
\hline $2928 \mathrm{H}$ & $2: 28: 24.9$ & $72: 34: 47.5$ & L1340A \\
\hline $2928 I$ & $2: 28: 28.5$ & $72: 34: 56.0$ & L1340A \\
\hline $2928 \mathrm{~J}$ & $2: 28: 29.7$ & $72: 34: 36.2$ & L1340A \\
\hline $2928 \mathrm{~K}$ & $2: 28: 35.6$ & $72: 34: 33.6$ & L1340A \\
\hline $2928 \mathrm{~L}$ & $2: 28: 39.6$ & $72: 34: 25.5$ & L1340A \\
\hline $2928 \mathrm{M}$ & $2: 28: 42.0$ & $72: 34: 17.1$ & L1340A \\
\hline $2928 \mathrm{~N}$ & $2: 28: 53.4$ & $72: 34: 11.5$ & L1340A \\
\hline 29280 & $2: 28: 56.9$ & $72: 34: 04.0$ & L1340A \\
\hline 2928P & $2: 29: 06.4$ & $72: 34: 07.8$ & L1340A \\
\hline MHO 2929 & $2: 27: 59.2$ & $72: 38: 21.2$ & L1340A \\
\hline MHO 2930 & 2:28:08.9 & $72: 36: 28.4$ & L1340A \\
\hline MHO 2931 & $2: 28: 15.0$ & $72: 36: 53.7$ & L1340A \\
\hline MHO 2932A & $2: 28: 15.6$ & $72: 37: 45.3$ & L1340A \\
\hline 2932B & $2: 28: 20.5$ & $72: 37: 41.9$ & L1340A \\
\hline MHO 2933 & $2: 28: 20.4$ & $72: 39: 24.6$ & L1340A \\
\hline MHO 2934 & $2: 28: 22.4$ & $72: 38: 56.5$ & L1340A \\
\hline MHO 2935 & $2: 28: 24.9$ & $72: 35: 24.7$ & L1340A \\
\hline MHO 2936 & $2: 28: 53.1$ & $72: 36: 12.1$ & L1340A \\
\hline MHO 2937A & $2: 29: 07.2$ & $72: 43: 42.3$ & L1340A \\
\hline 2937B & $2: 29: 08.6$ & $72: 43: 49.8$ & L1340A \\
\hline MHO 2938A & $2: 29: 29.8$ & $72: 37: 34.6$ & L1340A \\
\hline 2938B & $2: 29: 31.6$ & $72: 37: 41.2$ & L1340A \\
\hline MHO 2939A & $2: 29: 43.1$ & $72: 43: 53.9$ & L1340A \\
\hline 2939B & $2: 29: 42.4$ & $72: 44: 32.4$ & L1340A \\
\hline $2939 C$ & $2: 29: 37.8$ & $72: 44: 52.8$ & L1340A \\
\hline 2939D & $2: 29: 39.9$ & $72: 45: 05.6$ & L1340A \\
\hline 2939E & $2: 29: 40.4$ & $72: 45: 13.1$ & L1340A \\
\hline $2939 \mathrm{~F}$ & $2: 29: 35.2$ & $72: 46: 16.0$ & L1340A \\
\hline 2939G & $2: 29: 44.3$ & $72: 43: 45.8$ & L1340A \\
\hline $2939 \mathrm{H}$ & $2: 29: 45.4$ & $72: 43: 37.2$ & L1340A \\
\hline 2939I & $2: 29: 44.9$ & $72: 42: 15.6$ & L1340A \\
\hline MHO 2940 & $2: 30: 06.8$ & $72: 39: 54.1$ & L1340A \\
\hline MHO 2941 & $2: 26: 42.0$ & $72: 54: 24.4$ & L1340B \\
\hline MHO 2942A & $2: 27: 54.9$ & $72: 59: 25.1$ & L1340B \\
\hline 2942B & $2: 27: 49.0$ & $72: 59: 30.9$ & L1340B \\
\hline $2942 C$ & $2: 27: 45.4$ & $72: 59: 29.8$ & L1340B \\
\hline 2942D & $2: 27: 26.4$ & $72: 59: 27.4$ & L1340B \\
\hline $2942 \mathrm{E}$ & $2: 28: 08.5$ & $72: 59: 02.1$ & L1340B \\
\hline $2942 \mathrm{~F}$ & $2: 28: 17.1$ & $72: 58: 45.3$ & L1340B \\
\hline $2942 \mathrm{G}$ & $2: 28: 23.0$ & $72: 58: 28.1$ & L1340B \\
\hline $2942 \mathrm{H}$ & $2: 28: 23.8$ & $72: 58: 33.8$ & L1340B \\
\hline 2942I & $2: 28: 26.9$ & $72: 58: 28.2$ & L1340B \\
\hline 2942J & $2: 29: 12.9$ & $72: 57: 56.0$ & L1340B \\
\hline $2942 \mathrm{~K}$ & $2: 29: 13.5$ & $72: 57: 47.9$ & L1340B \\
\hline 2942L & $2: 29: 18.1$ & $72: 57: 44.1$ & L1340B \\
\hline $2942 \mathrm{M}$ & $2: 29: 27.7$ & $72: 57: 47.8$ & L1340B \\
\hline
\end{tabular}

Table 1

(Continued)

\begin{tabular}{|c|c|c|c|}
\hline MHO Designation & R.A. (J2000) & Decl. (J2000) & Region \\
\hline MHO 2943A & $2: 27: 55.7$ & 73:03:53.2 & L1340B \\
\hline 2943B & $2: 27: 51.6$ & 73:03:54.4 & L1340B \\
\hline $2943 C$ & $2: 28: 00.1$ & $73: 03: 54.6$ & L1340B \\
\hline 2943D & $2: 28: 04.1$ & 73:03:39.1 & L1340B \\
\hline MHO 2944 & $2: 29: 42.9$ & $72: 54: 21.3$ & L1340B \\
\hline MHO 2945 & $2: 30: 16.6$ & $72: 59: 05.2$ & L1340B \\
\hline MHO 2946A & $2: 30: 02.8$ & $73: 02: 51.9$ & L1340B \\
\hline 2946B & $2: 29: 58.5$ & 73:03:05.5 & L1340B \\
\hline 2946C & $2: 29: 30.2$ & $73: 03: 32.2$ & L1340B \\
\hline 2946D & $2: 30: 18.7$ & $73: 02: 45.5$ & L1340B \\
\hline $2946 \mathrm{E}$ & $2: 30: 31.2$ & $73: 02: 29.4$ & L1340B \\
\hline MHO 2947 & $2: 30: 44.9$ & $73: 02: 51.0$ & L1340B \\
\hline MHO 2948 & $2: 31: 13.3$ & 73:02:37.1 & L1340B \\
\hline MHO 2949 & $2: 30: 47.6$ & $72: 59: 30.2$ & L1340B \\
\hline MHO 2950 & $2: 30: 53.7$ & 72:59:07.8 & L1340B \\
\hline MHO 2951 & $2: 32: 14.3$ & $72: 56: 10.2$ & L1340B \\
\hline MHO 2952 & $2: 31: 30.4$ & $72: 39: 42.5$ & L1340C \\
\hline MHO 2953 & $2: 32: 21.7$ & 72:40:03.1 & L1340C \\
\hline MHO 2954 & $2: 32: 22.1$ & $72: 39: 49.0$ & L1340C \\
\hline MHO 2955 & $2: 32: 27.0$ & $72: 38: 26.7$ & L1340C \\
\hline MHO 2956 & $2: 32: 29.8$ & $72: 38: 38.1$ & L1340C \\
\hline MHO 2957 & $2: 32: 32.2$ & $72: 44: 14.1$ & L1340C \\
\hline MHO 2958 & $2: 32: 33.8$ & $72: 38: 22.2$ & L1340C \\
\hline MHO 2959 & $2: 32: 35.1$ & $72: 40: 32.1$ & L1340C \\
\hline MHO 2960 & $2: 32: 37.9$ & $72: 39: 41.0$ & L1340C \\
\hline MHO 2961 & $2: 32: 41.0$ & $72: 43: 33.7$ & L1340C \\
\hline MHO 2962 & $2: 32: 45.4$ & $72: 46: 56.1$ & L1340C \\
\hline MHO 2963 & $2: 32: 45.7$ & $72: 38: 18.6$ & L1340C \\
\hline MHO 2964A & $2: 33: 02.5$ & $72: 43: 31.2$ & L1340C \\
\hline 2964B & $2: 33: 02.3$ & $72: 43: 42.8$ & L1340C \\
\hline 2964C & $2: 33: 01.8$ & $72: 44: 03.4$ & L1340C \\
\hline 2964D & $2: 33: 04.3$ & $72: 43: 18.5$ & L1340C \\
\hline 2964E & $2: 33: 04.9$ & $72: 42: 46.0$ & L1340C \\
\hline $2964 \mathrm{~F}$ & $2: 33: 04.2$ & $72: 42: 20.0$ & L1340C \\
\hline MHO 2965 & 2:33:09.6 & $72: 48: 33.9$ & L1340C \\
\hline MHO 2966A & $2: 34: 06.7$ & $72: 43: 03.5$ & L1340C \\
\hline 2966B & $2: 34: 06.8$ & $72: 43: 15.5$ & L1340C \\
\hline 2966C & $2: 34: 08.2$ & $72: 42: 59.7$ & L1340C \\
\hline
\end{tabular}

\section{1. $L 1340 A$}

\subsubsection{A 3.7 pc Long Outflow Lobe: MHO 2925, MHO 2937, and HH 487}

MHO 2925 is a beautiful, filamentary shock system that outlines a bow shock shape (Figures 2 and 3). The apex of this bow shock is coincident with HH 487 A, discovered by Kumar et al. (2002). Based on the shock morphology, the outflow source must lie to the northeast somewhere along a line at a position angle of about $54^{\circ} \pm 1^{\circ}$.

The nearest candidate source along that line is 15.5 away. That star (SSTSL2 J022907.88+724347.2) was cataloged by Kun et al. (2016b) as a flat SED source. SSTSL2 J022907.88 +724347.2 is surrounded by a reflection nebula cataloged by Magakian et al. (2003) as their RN3. Our J, H, and $\mathrm{K}_{S}$ images also show a small reflection nebula surrounding the star (Figure 3). In the $\mathrm{J}$ image, the reflection nebula is a classic, double-lobed shape with an apparent disk shadow obscuring the star itself. At the $\mathrm{H}$ band, the star becomes visible, and the double-lobed structure of the reflection nebula is less apparent, though a loop-like structure is now visible in the northeast lobe 


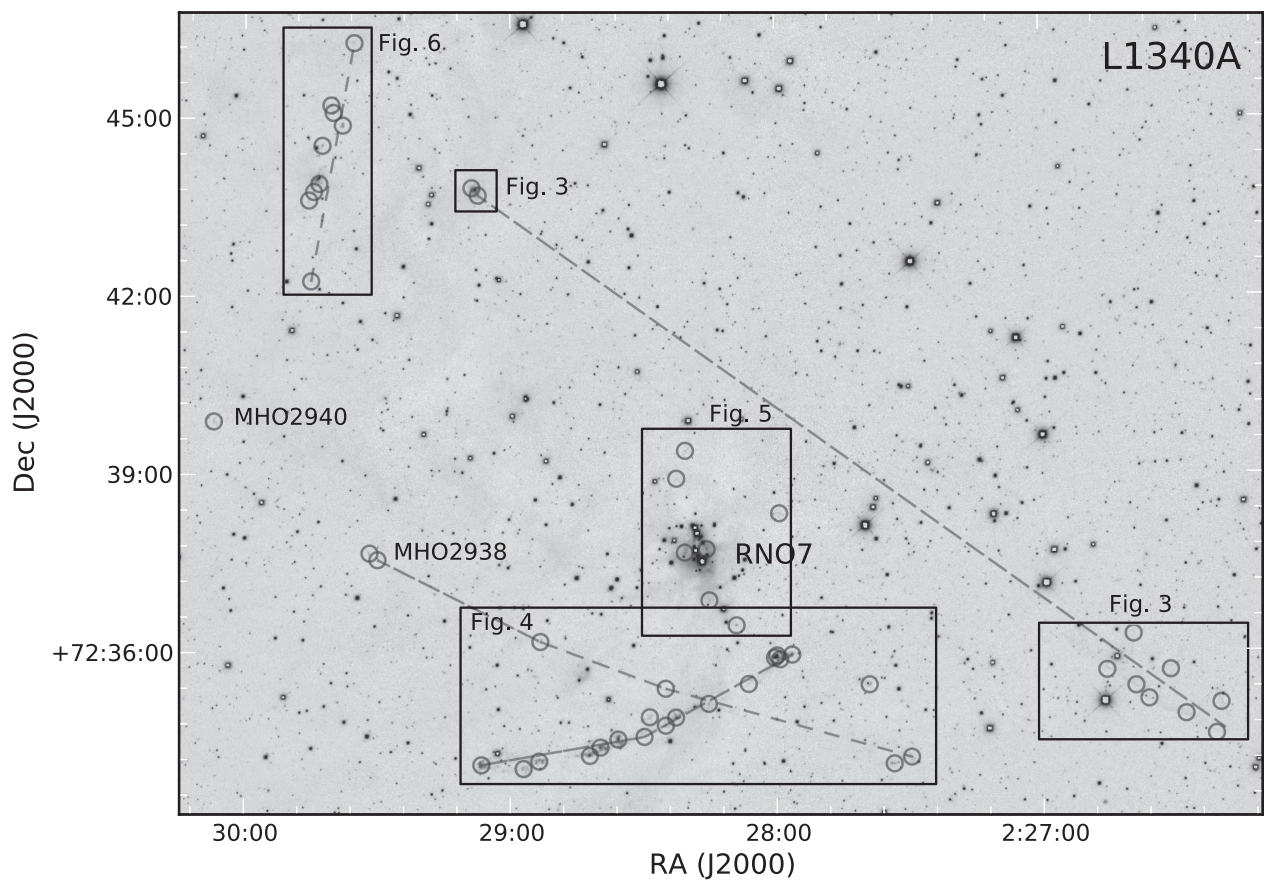

Figure 2. A $\mathrm{H}_{2}$ image of the $\mathrm{L} 1340 \mathrm{~A}$ region. Circles mark the positions of $\mathrm{H}_{2}$ shocks, and boxes indicate the locations of subsequent figures in the text.

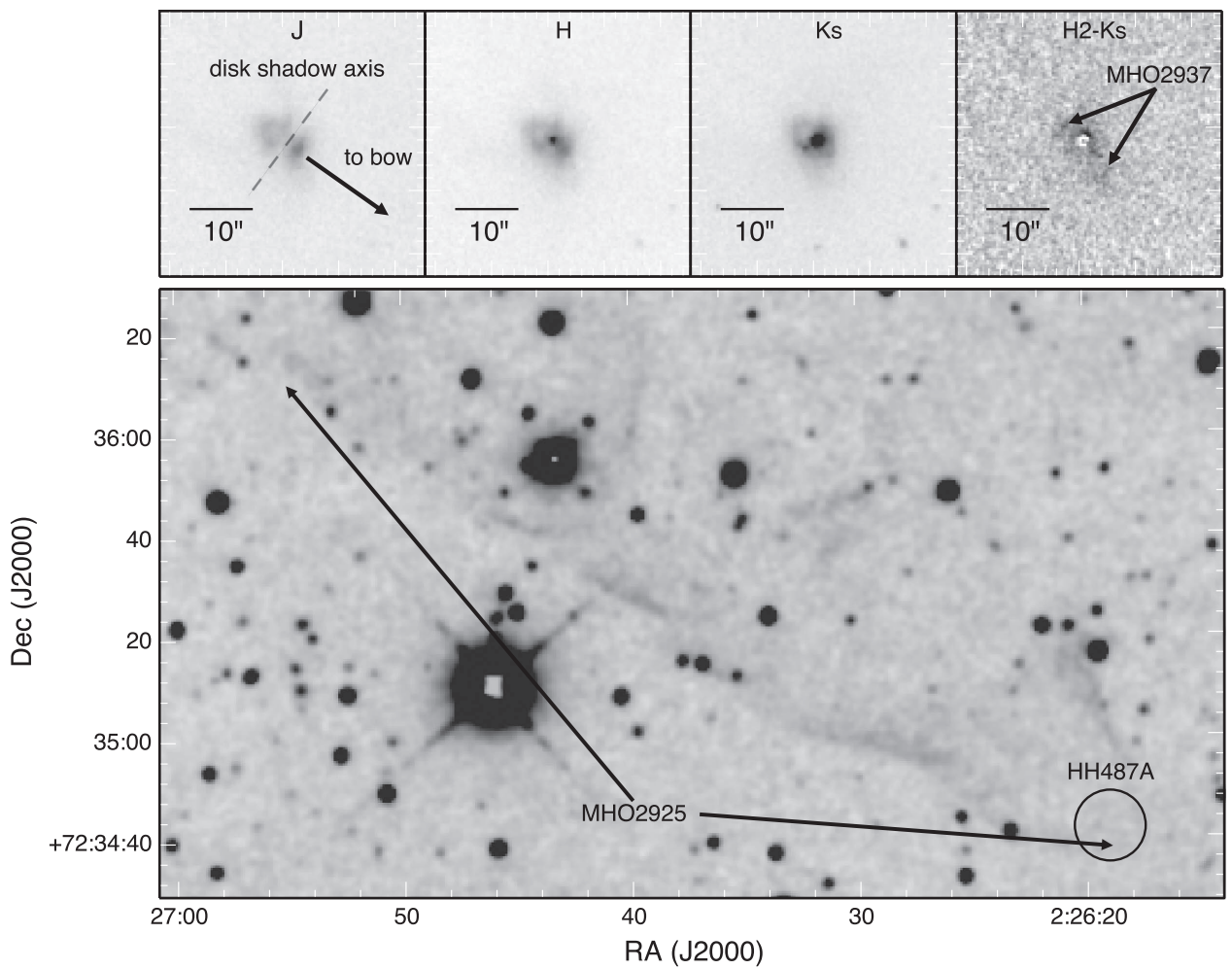

Figure 3. $\mathrm{A} \mathrm{H}_{2}$ image of the MHO 2925 bow shock discussed in Section 3.1.1. The upper panels show the region around SSTSL2 J022907.88+724347.2 in various filters, including a $\mathrm{H}_{2}-\mathrm{Ks}$ difference image that shows the MHO 2937 jet.

of the nebula. At $\mathrm{K}_{S}$, the star is much brighter, and the loop-like structure seen in the $\mathrm{H}$ band is still visible.

The disk shadow lies along a position angle of $143^{\circ} \pm 4^{\circ}$, which is perpendicular (within the measurement error) to the position angle defined by the vector from the star to MHO 2925.

In a $\mathrm{H}_{2}-\mathrm{K}_{S}$ difference image, there appears to be residual $\mathrm{H}_{2}$ emission around the star in a filamentary structure
(Figure 3). This filament is oriented at position angle $52^{\circ} \pm 6^{\circ}$ and extends from the northeast to the southwest across the star, in reasonable agreement with the direction to MHO 2925.

Based on the position of the source relative to the perceived flow axis of the MHO 2925 shock system, the angle of the disk shadow, and the angle of the MHO 2937 jet, we conclude that the HH 487 A, MHO 2925, and MHO 2937 shocks are driven 


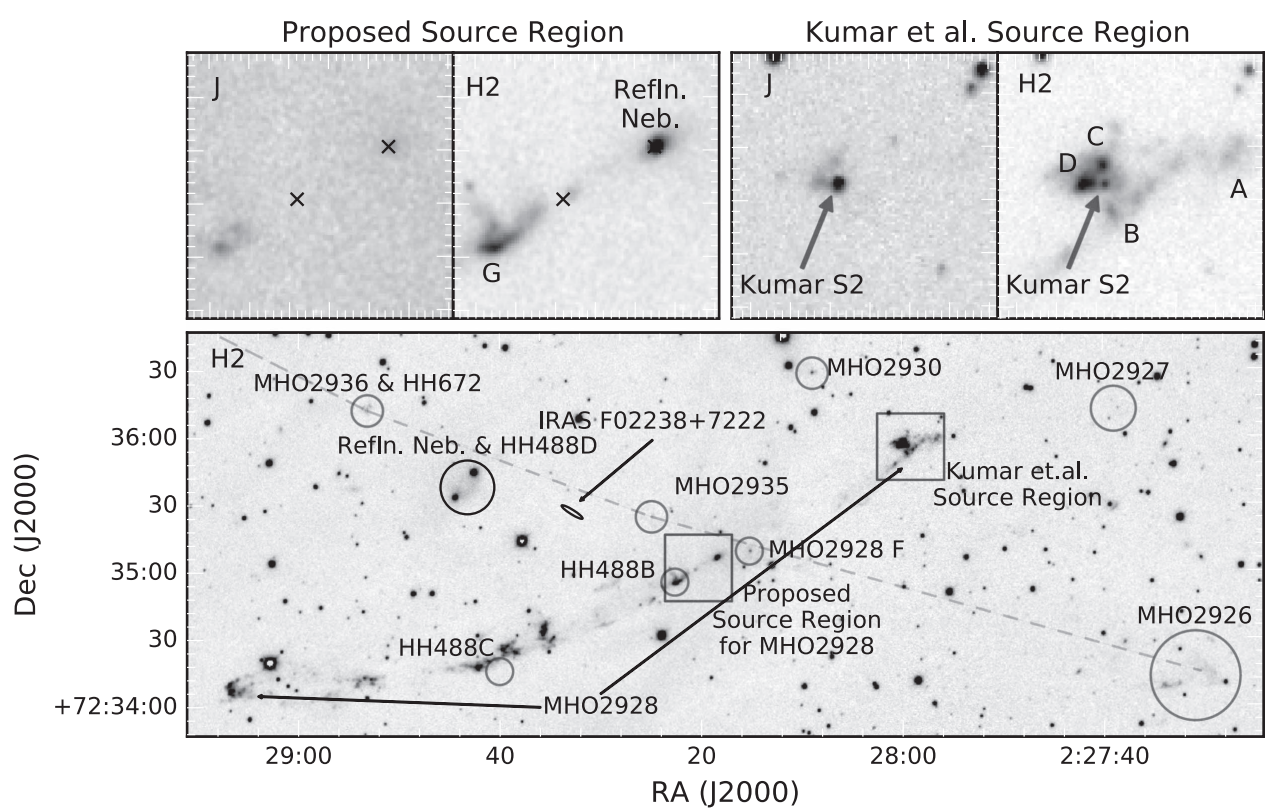

Figure 4. The $\mathrm{J}$ and $\mathrm{H}_{2}$ images of the region around MHO 2928. The insets along the top show close-up views of our proposed source region for MHO 2928 and the source region proposed by Kumar et al. (2002). In the insets of our proposed source region, the positions of SSTSL2 J022820.81+723500.5 or SSTSL2 J022820.81 +723500.5 are marked with $\mathrm{x}$, and MHO 2928 knot G and the reflection nebula discussed in the text are labeled. In the insets of the source region proposed by Kumar et al. (2002), MHO 2928 knots A to D are labeled. The Kumar et al. (2002) feature S1 is coincident with knot D. The Kumar et al. (2002) feature J1 is coincident with knot C. The Kumar et al. (2002) feature S2 is labeled. We find no strong $\mathrm{H}_{2}$ feature corresponding to the Kumar et al. (2002) feature J2.

by the SSTSL2 J022907.88+724347.2 source star. This makes the southwest lobe of this outflow 15.5 in length, which at a distance of $825 \mathrm{pc}$ corresponds to a length on the sky of $3.7 \mathrm{pc}$.

To the northeast of the source, we see some $\mathrm{H}_{2}$ shocks about 2.5 arcmin away near the same axis, but we associate those with the MHO 2939 flow from IRAS F02250+7230 (see Section 3.1.7), so we are unable to identify any distant components of the northeast lobe of the outflow.

The catalog position of the $\mathrm{A} 3 \mathrm{NH}_{3}$ core of Kun et al. (2003) lies $28^{\prime \prime}$ from the reflection nebula around SSTSL2 J022907.88 +724347.2 . This is in good positional agreement given that the half-power beam width and grid size of their map was $40^{\prime \prime}$.

Our association of MHO 2925 (HH 487) with SSTSL2 J022907.88+724347.2 is in disagreement with the conclusion by Kumar et al. (2002) and Kun et al. (2014) that the source of HH 487 is IRAS $02224+7227$, which lies roughly 6 arcmin away from the bow shock, but at a position angle of $28^{\circ} .4$. We believe that the morphology of the $\mathrm{H}_{2}$ bow shock is inconsistent with their interpretation. Kun et al. (2014) discuss the dynamic age of HH 487 if the source were IRAS 02224 +7227 , but our association of that shock with SSTSL2 J022907.88+724347.2 places it 2.5 times as far away, and thus the dynamic age of the outflow would be much larger than the 6,500 years they estimate.

\subsubsection{A Bright, Curved Outflow: MHO 2928, HH 488 B, HH 488 C}

This flow is a long chain of $\mathrm{H}_{2}$ knots and filaments that is about 5.7 arcmin long (Figure 4), which corresponds to a length of about $1.4 \mathrm{pc}$ at an assumed distance of $825 \mathrm{pc}$. In this chain of $\mathrm{H}_{2}$ knots extending to the southeast, we find knots that correspond to HH $488 \mathrm{~B}$ and $\mathrm{HH} 488 \mathrm{C}$ of Kumar et al. (2002). Kumar et al. (2002) also identified HH 488 D, which lies $\sim 1$ !3 north of the chain of $\mathrm{H}_{2}$ knots. We believe this shock is not associated with this flow, and it is discussed elsewhere in this paper (see Section 3.1.3).
The gradual curve of the shock system suggests that either the source is moving to the southwest or that the source star is precessing and thus changing the launch angle of the flow.

Kumar et al. (2002) suggested that the flow was launched from a star that corresponds to SSTSL2 J022759.92 +723556.4, a probable double star. Kun et al. (2016b) catalog it as a flat SED source, but note that the SED "results from the composite fluxes of the central objects." This region is very complex, and Kumar et al. (2002) identified two candidate jets in $\mathrm{H} \alpha$ and [S II] emerging from two stars (which they designated S1 and S2) separated by about 2 arcsec at position angles of $320^{\circ}$ and $275^{\circ}$, respectively. S1 is the brighter of the two in $\mathrm{H} \alpha$, but $\mathrm{S} 2$ is the only one visible in their continuum (Gunn z) image.

In our broadband J (see Figure 4) and $\mathrm{H}$ (not shown) images, there are five objects within 8 arcsec of the Kumar source. Two of these are 2.7 arcsec to the east and 2.0 arcsec to the north, respectively, and there appears to be extended emission connecting these two points to the source star. The arrangement of these three sources appears to match the arrangement of the Kumar et al. (2002) sources S1 and S2 and J1 jet; however, the positions of S1 and S2 listed in Kumar et al. (2002) lie about 1.5 arcsec north of the corresponding objects in our images. This may be due to an offset in the world coordinate system in the Kumar et al. (2002) coordinates. For this discussion we will assume that the Kumar et al. (2002) sources correspond to the objects visible in our images. In this case, S2 of Kumar et al. (2002) is clearly a stellar component in our images.

Whether the other two objects in our images are stars, reflection nebulae, or shocks is not completely clear. The source corresponding to the Kumar et al. (2002) S1, however, is very bright in $\mathrm{H}_{2}$, so we suspect that it is shocked gas rather than a stellar source, so we consider it a $\mathrm{H}_{2}$ shock (MHO 2928 D). The object to the northwest (corresponding to Kumar et al. $2002 \mathrm{~J} 1$ ) is also very bright in $\mathrm{H}_{2}$, so we similarly consider it a $\mathrm{H}_{2}$ shock (MHO $2928 \mathrm{C}$ ). We see no 
object corresponding to the Kumar et al. (2002) object J2, which lies 3 arcsec due west of the Kumar et al. (2002) S2.

In the $\mathrm{H}_{2}$ filter, a filamentary chain of knots (MHO $2928 \mathrm{~B}$ ) is visible about 4 arcsec southwest of the Kumar source (Figure 4). The continuous part of the filament is about 23 arcsec long along a position angle of $139^{\circ}$. This filament does not pass through the Kumar source star, so it is unlikely that this is a jet, but it is possibly the shocked wall of the outflow cavity. It should be noted that this filament does not correspond to the jet features in Kumar et al. (2002) as it lies south of the source star, while the Kumar et al. (2002) features are coincident with the star.

After inspection of the large-scale structure of the outflow and of the WISE 3.4, 4.6, 12, and $22 \mu \mathrm{m}$ images, we conclude that the source of the flow is not in the region suggested by Kumar et al. (2002), but that it lies 1!6-1!8 to the southeast along the flow axis and that the flow is driven by either SSTSL2 J022820.81+723500.5 or SSTSL2 J022818.51 +723506.2 . We prefer this as the source region (similar to the conclusion of Kun et al. 2016b) instead of that suggested by Kumar et al. (2002) because these two candidate source stars were both classified as Class 0/I by Kun et al. (2016b), and SSTSL2 J022820.81+723500.5 was discussed as a candidate Class 0 source. In addition, the filamentary $\mathrm{H}_{2}$ structure around these two sources (MHO 2928 G) passes through the sources themselves and could plausibly be a jet, unlike the filament (MHO 2928 B) south of the Kumar et al. (2002) source star. Lastly, these two sources are coincident with the A1 $\mathrm{NH}_{3}$ core of Kun et al. (2003). Of the two sources, SSTSL2 J022818.51 +723506.2 is coincident with a reflection nebula visible in our $\mathrm{J}$, $\mathrm{H}$, and $\mathrm{K}_{S}$ images (see inset in Figure 4), while SSTSL2 J022820.81+723500.5 is invisible in $\mathrm{J}$ and $\mathrm{H}$ and only faintly visible in $\mathrm{K}_{S}$ and $\mathrm{H}_{2}$.

\subsubsection{A Candidate Large-scale Outflow: $M H O 2926$, MHO 2935, MHO 2936, MHO 2938, MHO 2940, HH 488 D, HH 672}

MHO 2926 (Figure 4) is a complex of knots that makes up a nice bow shock pointing roughly back to a source that must lie along PA $\sim 60^{\circ}-80^{\circ}$. Along that line lie HH $488 \mathrm{D}$ and HH 672 in addition to several $\mathrm{H}_{2}$ shocks (MHO 2928, 2935, 2936, 2938, 2940).

MHO 2935 (Figure 4) is a compact $\mathrm{H}_{2}$ knot. HH 488 D has no clear $\mathrm{H}_{2}$ counterpart, but it is coincident with the reflection nebulosity visible in our $\mathrm{J}, \mathrm{H}$, and $\mathrm{K}_{S}$ images. $\mathrm{HH} 672$ has a $\mathrm{H}_{2}$ counterpart (MHO 2936; Figure 4), which is a small cluster of $\mathrm{H}_{2}$ knots. MHO 2938 (Figure 2) is a pair of small knots separated by about $12^{\prime \prime}$.

While the association of these features is not conclusive, we find it likely that there is a flow along PA $\sim 75^{\circ}$, emanating from one of the four candidate sources described below. This flow would be composed of MHO 2926, MHO 2935, HH 488 D, MHO 2936 (and its optical counterpart HH 672), and MHO 2938 (Figure 2) with the likelihood that some of the $\mathrm{H}_{2}$ emission in MHO 2928 is also part of this flow where the two flows cross. If this is the case, this flow is 9!9 long, which would correspond to $2.4 \mathrm{pc}$. In addition, this flow has the shape of a gentle arc similar to the MHO 2928 flow. The opening of the arc faces back toward RNO7. If the arc is due to the source being in motion, then this star could potentially have been dynamically ejected from the cluster core.

The knot MHO 2940 (Figure 2) lies another 3.4 arcmin northeast of MHO 2938 along the flow axis. It may be a distant shock in this flow, but the association is unclear because it also lies near the axis of the MHO 2939 flow.

There are several candidate sources that lie near the axis of this flow. From east to west, they are SSTSL2 J022844.40 $+723533.5, \quad$ SSTSL2 J022842.57+723544.3, IRAS F02238 +7222, and WISE J022817.97+723517.5. We briefly discuss each in the paragraphs below.

The reflection nebula coincident with HH 488 D (Figure 4) spans a region between two stars. The southern star is SSTSL2 J022844.40+723533.5, and the northern is SSTSL2 J022842.57+723544.3. Both were classified by Kun et al. (2016b) as Class 0/I sources based on their SED slopes.

IRAS F02238+7222 (identified by Kumar et al. 2002 as a candidate young star) also lies along the axis of the flow, but we find no sources in the WISE images or in our near-IR images that seem to correspond to IRAS F02238+7222. We conclude that this is likely a background extragalactic source.

Lastly, there is WISE J022817.97+723517.5, a star near the intersection of the MHO 2928 flow and this flow's axis. This star was not selected as a protostar in Kun et al. (2016b) and lies somewhat outside the color parameters that Koenig \& Liesawitz (2014) use to classify WISE sources as protostars. However, it is visible in our $\mathrm{K}_{S}$ image and appears to have a faint filament of $\mathrm{H}_{2}$ emission (MHO 2928 F) that passes through the star along roughly the same axis that the flow is expected to occupy. Because this filament overlaps with MHO 2928 (and is cataloged as part of that MHO shock complex), it is impossible to tell if it is part of this flow or not, but the morphology is suggestive. WISE J022817.97 +723517.5 also lies within the contours of the $\mathrm{A} 1 \mathrm{NH}_{3}$ core of Kun et al. (2003). Although this core likely contains several stars as the source of the MHO 2928 flow (described in Section 3.1.2) is also present in this core. Because of the confused nature of the emission in this region (much of it presumably from the MHO 2928 flow), the coincidence of the WISE star and a shock filament is not as compelling as it would be in other regions, so we favor one of the two SSTSL sources mentioned above as the source for this flow.

\subsubsection{MHO 2932, HH 671 A}

Magakian et al. (2003) found two HH knots in the RNO7 region (HH $671 \mathrm{~A}$ and $\mathrm{B}$ ) that are separated by about 1 arcmin. Both objects have bright, compact $\mathrm{H}_{2}$ counterparts.

The counterpart to HH671 A (MHO 2932; Figure 5) lies 11 !" 2 west of one of the brighter stars in the RNO7 cluster. There is an additional knot (MHO 2932 B) that lies 10." 6 east of the same star. Their positions opposite one another across the star suggest that they may be the east and west components of an outflow that is launched from that star at a position angle of $99^{\circ} \pm 2^{\circ}$. The source star was cataloged as a $\mathrm{H} \alpha$ emission line star by both Magakian et al. (2003) (who designated it $\mathrm{H} \alpha$ star number 8) and Kun et al. (2016a) (star 19 in their Table 2).

The association of these shocks with a particular source star is tenuous at best due to the crowded field (several other $\mathrm{H} \alpha$ emission line stars lie in the RNO7 cluster) and to the lack of resolved morphology in the MHO objects. It should be noted this is in contradiction with the connection by Magakian et al. (2003) of HH 671 A and HH 671 B into a single flow, which is implied by giving them both the same $\mathrm{HH}$ number (HH 671) and distinguishing them by using the knot $\mathrm{A}$ and knot $\mathrm{B}$ designations. 


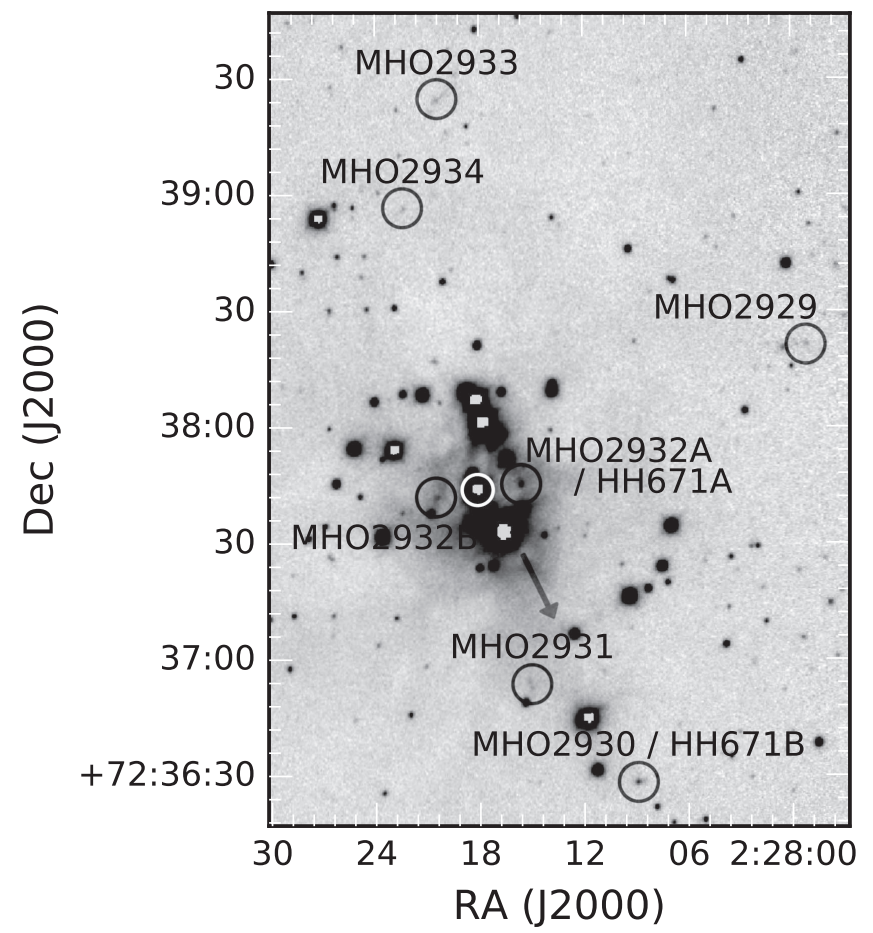

Figure 5. $\mathrm{A} \mathrm{H}_{2}$ image of the region around $\mathrm{MHO} 2932$ discussed in Section 3.1.4. The white circle indicates the position of WISE J022818.07 +723743.8 (Magakian et al. $2003 \mathrm{H} \alpha$ star number 8). The gray arrow indicates the approximate position and orientation of the jet discussed in Kumar et al. (2002) Section 3.1.

\subsubsection{Additional Shocks near the RNO7 Cluster: $M H O 2929$, MHO 2930, MHO 2933, MHO 2933, MHO 2934}

Five additional shocks lie within $\sim 1$ ! 6 of the RNO7 cluster (Figure 5). To the south lie MHO 2930 and MHO 2931. MHO 2930 is the counterpart to HH $671 \mathrm{~B}$. It is a bright, compact knot of $\mathrm{H}_{2}$ emission, which lies about 1 '.5 south of the center of the RNO7 cluster. MHO 2931 is a fainter, more diffuse feature that lies closer to the cluster $(1 ! 0$ south of RNO7).

To the north of RNO7 lie MHO 2934 and MHO 2933 at distances of 1!.2 and 1!6, respectively. MHO 2934 is a small, faint, compact knot, while MHO 2933 is a $12^{\prime \prime}$ long filament, oriented roughly northwest-southeast.

These four objects may make up the northern and southern components of one or more flows emerging from one of the stars in the RNO7 cluster, but a clear association with a particular flow or source star is not possible with these images.

MHO 2929, which lies 1!5 west of RNO7, consists of a single compact $\mathrm{H}_{2}$ knot.

Kumar et al. (2002) describe a candidate $\mathrm{HH}$ jet in this region. Their Figure 2(b) labels it as emerging from a $\mathrm{H} \alpha$ emission line star (labeled Hal in their Figure 2(c)). The feature is roughly 10 arcsec long, is stronger in [S II] than $\mathrm{H} \alpha$, and is oriented to the southwest. It points generally in the direction of MHO 2930 and some bright knots in MHO 2928, MHO 2927, and MHO 2926. However, we do not associate it with any of those as the alignments are not convincing, and we cannot confirm the existence of this jet as it does not show up in our near-IR images (Figure 5).

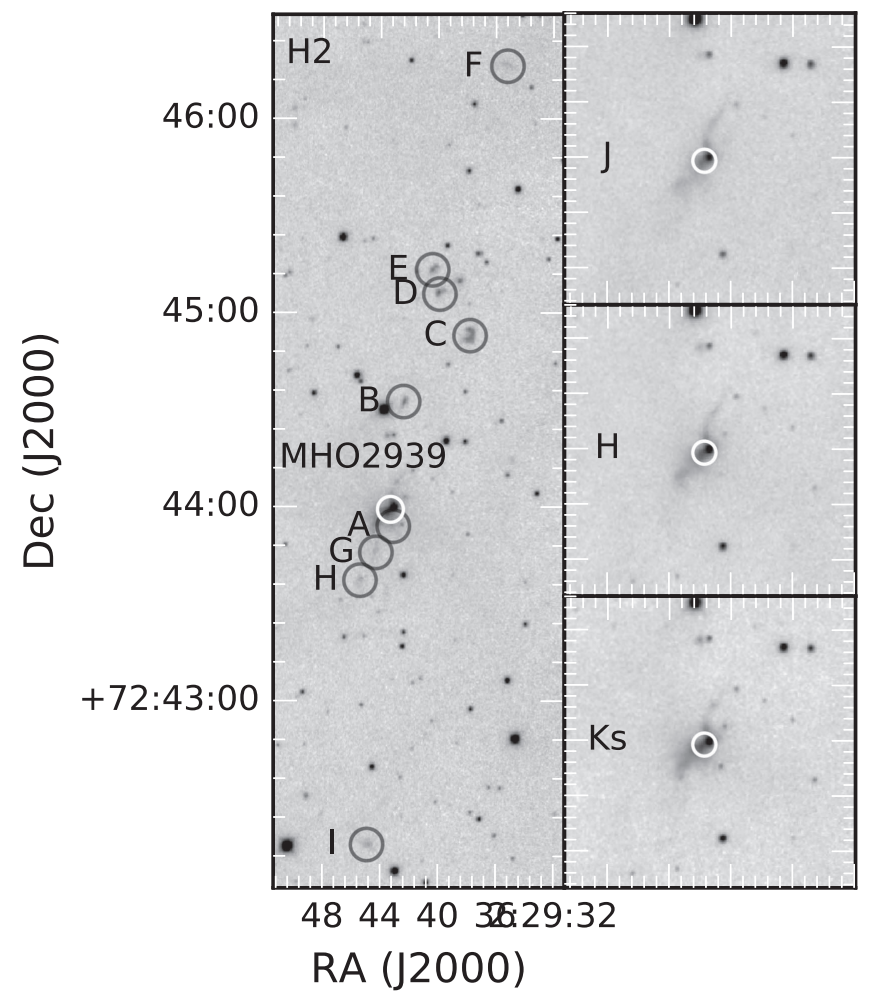

Figure 6. Set of $\mathrm{H}_{2}, \mathrm{~J}, \mathrm{H}$, and $\mathrm{K}_{S}$ images of the region around MHO 2939 discussed in Section 3.1.7. A white circle indicates the position of the binary source discussed in the text.

\subsection{6. $M H O 2927$}

MHO 2927 (Figure 4) lies about 3.7 arcmin southwest of RNO7. It has the appearance of a filament, about 9 arcsec long oriented along $\mathrm{PA} \sim 60^{\circ}$ with a brighter, possibly bow-shaped structure on the west end. A line drawn along $\mathrm{PA} \sim 60^{\circ}$ extending to the northeast away from the bow-shock-like structure passes near the southern edge of the RNO7 cluster of stars, suggesting that MHO 2927 may be launched by a source in that region, but the shock is faint, making the morphological link of this with any particular source tenuous at best. In addition, three nearby flat-spectrum SED protostars identified by Kun et al. (2016b) lie closer to this shock than RNO7 at position angles ranging from 75 to 85 .

\subsection{7. $\mathrm{MHO} 2939$, HH 489}

Kumar et al. (2002) found two knots (HH 489 A and B) that lie roughly symmetrically 1 arcmin on either side of an IRAS source. Kumar et al. (2002) also found a "wisp of nebulosity seen in Ha emission [which] extends out from both sides of the source along the flow direction." In our continuum $\left(\mathrm{J}, \mathrm{H}, \mathrm{K}_{S}\right.$ ) images (Figure 6), a filamentary reflection nebula is visible around the source along a position angle similar to that of the filament described by Kumar et al. (2002).

The IRAS source corresponds to a pair of Class I sources identified by Kun et al. (2016b) as SSTSL2 J022943.01 +724359.6 and SSTSL2 J022943.64+724358.6, which are separated by 2 ". 8 . They lie within the A4 $\mathrm{NH}_{3}$ core of Kun et al. (2003). One of these sources is presumably the driving source of the HH 489 flow.

Our $\mathrm{H}_{2}$ image (Figure 6) reveals several shocks in the region (designated MHO 2939) distributed along a line that 


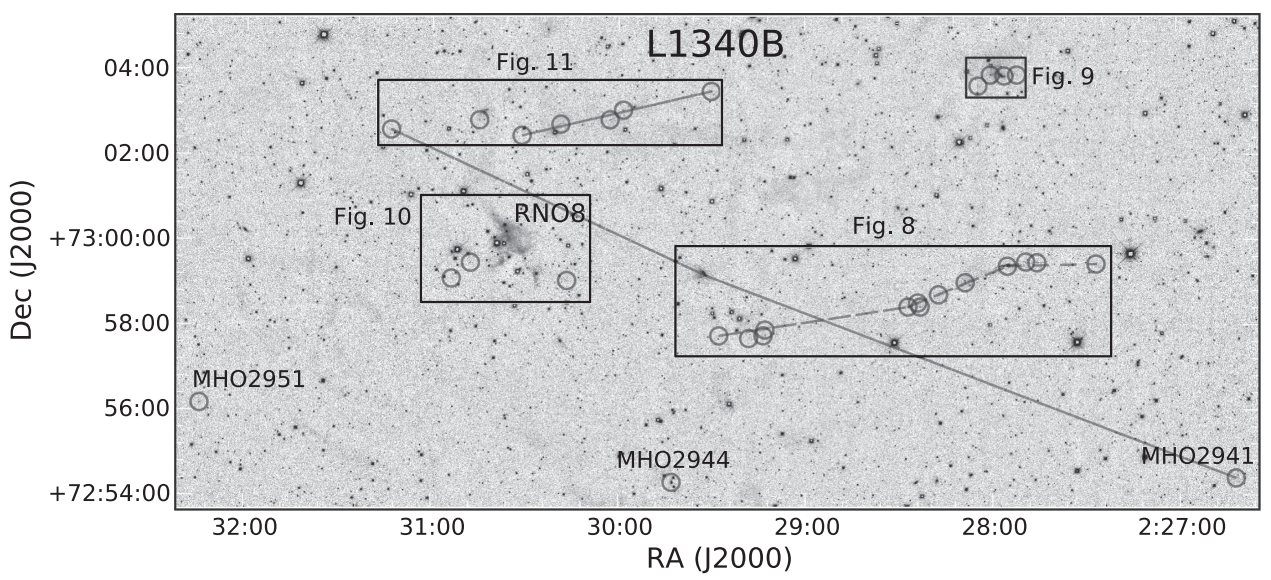

Figure 7. $\mathrm{A}_{2}$ image of the $\mathrm{L} 1340 \mathrm{~B}$ region. Circles mark the positions of $\mathrm{H}_{2}$ shocks, and boxes indicate the locations of subsequent figures in the text.

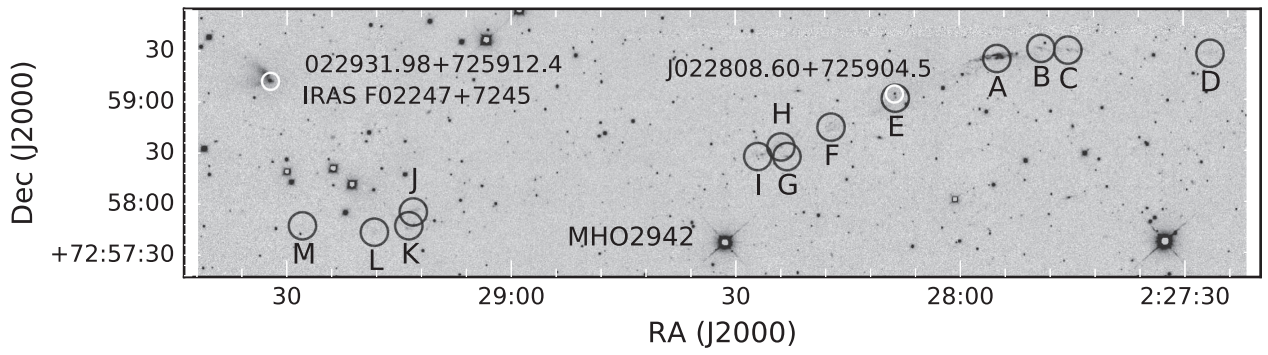

Figure 8. $\mathrm{A} \mathrm{H}_{2}$ image of the MHO $2942 \mathrm{~S}$-shaped outflow discussed in Section 3.2.1. The V-shaped reflection nebula around IRAS F02247+7245 (discussed in Section 3.2.2) is visible at the upper left corner of the image. The error ellipse for IRAS F02247+7245 is also marked in black. The SSTSL2 sources discussed in the text are marked with white circles and labeled.

corresponds to the position angle of the filament described by Kumar et al. (2002) and the filament visible in the $\mathbf{J}, \mathrm{H}$, and $\mathrm{K}_{S}$ images. Our shocks trace an outflow that is 4 arcmin long at a position angle of $\sim 170^{\circ}$.

The knot MHO 2940 lies not far off of this axis, about 4.5 arcmin south of the source along a position angle of $157^{\circ}$. However, it also lies along the axis of the MHO 2926 flow, so it is not clear which flow it is associated with.

\section{2. $L 1340 B$}

\subsubsection{A Large S-shaped Outflow: MHO 2942}

MHO 2942 (Figures 7 and 8) is a 9! 1 long chain of $\mathrm{H}_{2}$ knots orignally discovered in our 2005/2006 NICFPS run at Apache Point. The chain is oriented roughly east-west with a gentle S-shaped curve. Kun et al. (2016b) found a candidate Class 0 source (SSTSL2 J022808.60+725904.5) near this flow axis, 2." 6 north of the brightest condensation in knot E.

\subsubsection{The IRAS F02247+7245 Outflow: $M H O 2941$}

About 1!4 north of the easternmost knots in MHO 2942 lies a dramatic $\mathrm{V}$-shaped reflection nebula (visible in $\mathrm{H}_{2}$ in the upper left corner of Figure 8) with a star at its apex. The reflection nebula is visible at $\mathrm{J}, \mathrm{H}$, and $\mathrm{K}_{S}$, but the star (SSTSL2 J022931.98+725912.4) is only apparent in the $\mathrm{K}_{S}$ image. IRAS F02247+7245 lies 15 arcsec away from the star at the apex of the reflection nebula on a position angle of $54^{\circ}$. The uncertainty ellipse of the IRAS source is 14 arcsec along the major axis along a position angle of $58^{\circ}$. This places the Kun et al. (2016b) source just outside of the error ellipse, but we believe that the IRAS source corresponds to the Kun et al. (2016b) source, which they identified as a candidate Class 0 source.

The V-shaped reflection nebula opens to the east and is bright on the north and south edges and dark along its central axis, which lies along a position angle of roughly $70^{\circ}$. Roughly $13 ! 3$ to the southwest, opposite the opening of the reflection nebula (along a position angle of $249^{\circ}$ ), lies MHO 2941, which is a faint bow-shaped arc of $\mathrm{H}_{2}$ emission. Based on the agreement in position angle with the opening of the reflection nebula, we find it likely that MHO 2941 is a distant bow shock in a flow driven by IRAS F02247+7245. This means that one lobe of this flow is $13 ! 3(3.2 \mathrm{pc})$ long.

To the northeast, 8.1 arcmin away along a position angle of $65^{\circ}$, lies MHO 2948. The alignment of this shock relative to the axis of the reflection nebula makes the association of this shock with the IRAS F02247+7245 source compelling. On the other hand, this shock lies near the axis of, and may be part of, the MHO 2946 flow (see Figures 7 and 11).

Kun et al. (2016b) also describe a "jet-like feature, bright at $4.5 \mu \mathrm{m}$ on the western side (Figure 20)." By inspection of their Figure 20, we see that this jet-like feature emerges at a position angle of roughly $275^{\circ}$. This does not align with a line drawn to MHO 2948, and we find no shocked emission in that direction other than MHO 2942, which is clearly associated with a different source star.

We hypothesize that the jet-like feature in the Spitzer image is not a jet but is the wall of the outflow cavity. While the eastern cavity walls are visible in our $\mathrm{J}, \mathrm{H}$, and $\mathrm{K}_{S}$ images (see Figure 8), there is no corresponding western cavity. We suggest that this is due to greater extinction on that half of the outflow, either due to a local feature in the cloud structure or due to the ouflow being aimed toward Earth (i.e., we predict it is a 


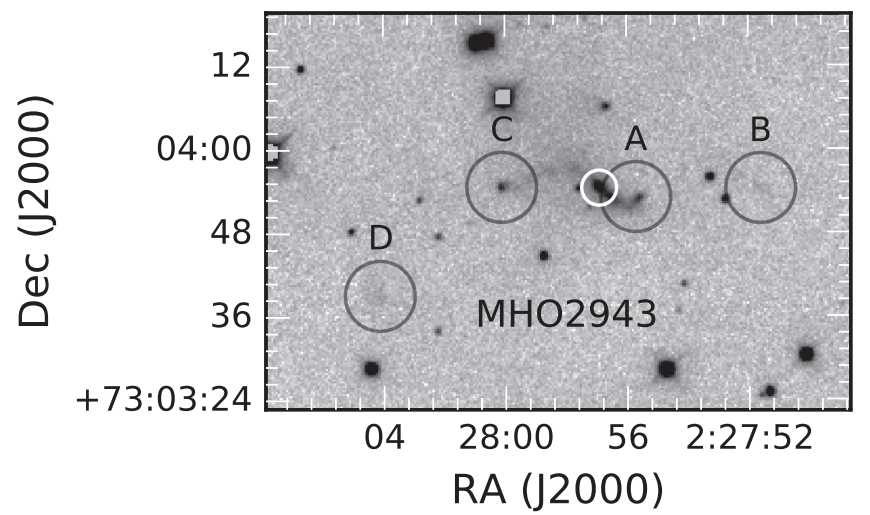

Figure 9. $\mathrm{A} \mathrm{H}_{2}$ image of the MHO 2943 flow discussed in Section 3.2.3. The source star (SSTSL2 J022756.91+730354.4) lies in the brightest portion of the reflection nebula. The $\mathrm{H}_{2}$ shocks are marked, and the position of the WISE source discussed in the text is indicated with a white circle.

blueshifted outflow lobe) while the western lobe is more highly extincted. Indeed, Kun et al. (2016b) note the "color difference between the eastern and western nebulosities," which in their Figure 20 can be seen as this feature being brighter in green $(4.5 \mu \mathrm{m})$ than in the blue $(3.6 \mu \mathrm{m})$. This could easily be due to a difference in extinction along the line of sight to the two cavity walls.

\subsubsection{A Compact S-shaped Outflow: MHO 2943}

MHO 2943 (Figure 9) is a set of four $\mathrm{H}_{2}$ knots that appears to emerge from the region surrounding SSTSL2 J022756.91 +730354.4, which Kun et al. (2016b) identified as a Class 0/I source. The star is visible in our $\mathrm{K}_{S}$ and $\mathrm{H}$ images but disappears in J. It is surrounded by a filamentary S-shaped reflection nebula about 20 arcsec across.

Two of the four MHO 2943 knots are very compact and bright and lie nearest the source, one 6.4 arcsec to the west, the other 14 arcsec to the east. The other two knots are fainter, more diffuse, and lie $24 \operatorname{arcsec}$ to the west and $36 \operatorname{arcsec}$ to the east, respectively.

\subsubsection{The RNO8 Region: MHO 2945, MHO 2949, MHO 2950}

Three $\mathrm{H}_{2}$ knots lie in a 1.4 arcmin diameter region roughly centered on the southern half of the RNO8 reflection nebula. All three knots are relatively faint. MHO 2945 and MHO 2950 are diffuse, while MHO 2949 is a compact knot (Figure 10). The MHO 2945 and MHO 2949 knots lie nearly equidistant $\left(70^{\prime \prime}\right.$ and $67^{\prime \prime}$, respectively) on either side of SSTSL2 J023032.44+725918.0, suggesting that they may be launched from this star, but this may be a chance alignment. Kun et al. (2016b) classified SSTSL2 J023032.44+725918.0 as a Class 0/I source, but they also describe its classification as ambiguous and declare that it falls "into the Class II regime near the Class I/ Class II boundary." Kun et al. (2016a) found it to be a "late $G$ spectral type with the Balmer lines in emission" (star 43 in their Table 2) and argue that it is a star surrounded by a disk seen at high inclination (leading to the relatively low extinction to the stellar photosphere).

\subsubsection{MHO 2946}

MHO 2946 (Figure 11) is a chain of $\mathrm{H}_{2}$ knots about 4.5 arcmin long. The axis defined by the knots passes near three candidate source stars.

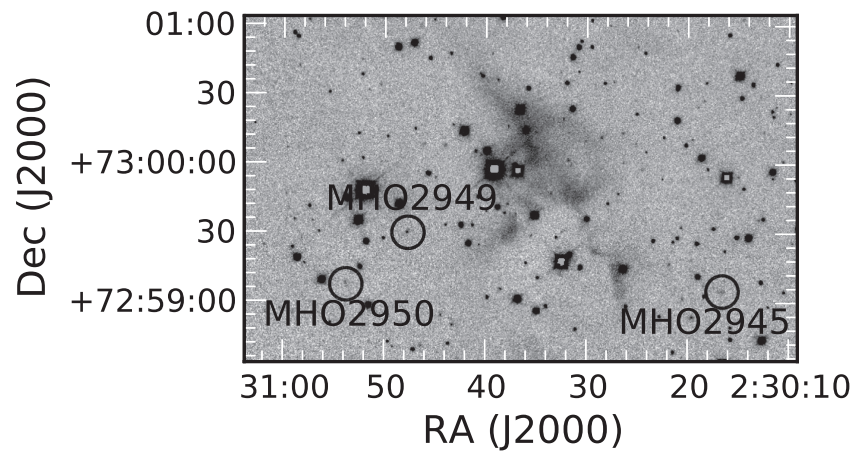

Figure 10. $\mathrm{A} \mathrm{H}_{2}$ image of the RNO8 region discussed in Section 3.2.4 showing the locations of MHO 2945, MHO 2949, and MHO 2950.

The western of these candidates is SSTSL2 J023042.36 +730305.1, a Class 0/I star identified by Kun et al. (2016b). This star has a small, faint wisp of $\mathrm{K}_{S}$ emission, which may be a reflection nebula emerging on the western side of the star (see also Section 3.2.6). This source star lies farthest from the apparent axis of MHO 2946, so we do not favor it as a source candidate.

Another candidate source is SSTSL2 J023020.61+730233.7, which Kun et al. (2016b) cataloged as a flat-spectrum source. This star is bright at all near-IR wavelengths $\left(\mathrm{J}, \mathrm{H}, \mathrm{K}_{S}\right)$ and is even visible faintly in the POSS R image, though not in the POSS B image. IRAS F02256+7249 lies about 18 arcsec from this second candidate star. The eastern candidate source star is SSTSL2 J022955.10+730309.1, another Class 0/I star in the Kun et al. (2016b) catalog. The B1 $\mathrm{NH}_{3}$ core of Kun et al. (2003) lies roughly in between these two more eastern sources.

\subsection{6. МHO 2947}

Roughly 40 arcsec northeast of the easternmost component of MHO 2946 lies a fan-shaped reflection nebula with a star (SSTSL2 J023042.36+730305.1) at its apex (see also Section 3.2.5). The B2 $\mathrm{NH}_{3}$ core of Kun et al. (2003) lies 0.5 northwest of the apex of the reflection nebula. The nebula opens toward position angle $\sim 120$ and is visible in all of our $\mathrm{J}$, $\mathrm{H}$, and $\mathrm{K}_{S}$ images, while the star is invisible at $\mathrm{J}$ and $\mathrm{H}$ but bright in $\mathrm{K}_{S}$. About 18 arcsec southeast of the star along $\mathrm{PA} \sim 140^{\circ}$ lies a faint, diffuse $\mathrm{H}_{2}$ knot (MHO 2947), which is likely driven by the source embedded in that reflection nebula. It is also possible, however, that this is a distant shock in the MHO 2946 flow.

\subsubsection{Distant $H_{2}$ Shocks: $M H O$ 2951, MHO 2944}

Two shocks in the L1340 B region lie far from the other groupings. MHO 2944 (Figure 7) lies in the southern part of this region. It is a bright, compact $\mathrm{H}_{2}$ knot that lies roughly equidistant from two Class 0/I sources identified by Kun et al. (2016b): SSTSL2 J022932.31+725503.2 (also H $\alpha$ emission line star number 38 in Kun et al. (2016a) Table 2) and SSTSL2 J022949.62+725326.1.

MHO 2951 is a faint, diffuse knot (Figure 7) that lies about 3 arcmin southeast of four sources identified as either Class 0/I or flat SED by Kun et al. (2016b). 


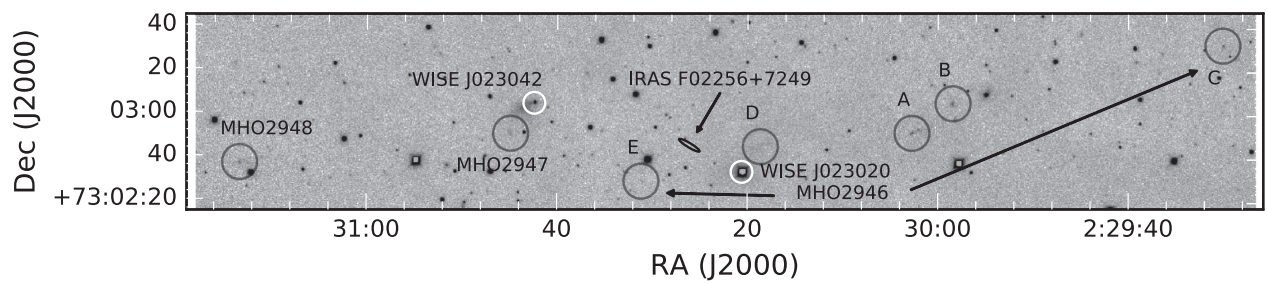

Figure 11. $\mathrm{A} \mathrm{H}_{2}$ image of the MHO 2946 flow discussed in Section 3.2.5. The position of IRAS F02256+7249 is indicated with an ellipse that corresponds to the positional error of the source in the IRAS catalog. The reflection nebula surrounding SSTSL2 J023042.36+730305.1 is visible in the left half of the image. The positions of SSTSL2 J023020.61+730233.7 and SSTSL2 J022955.10+730309.1 are indicated with white circles.

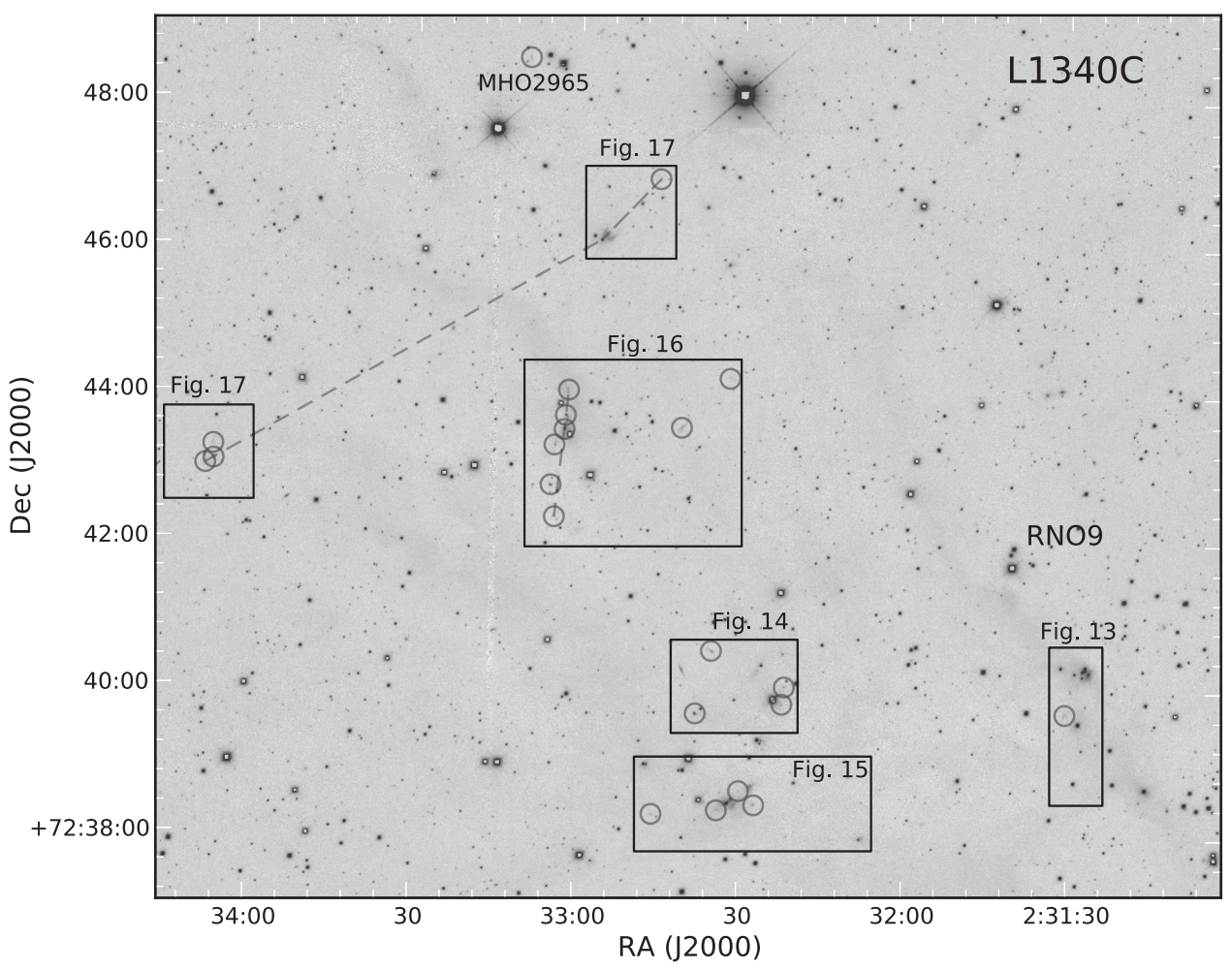

Figure 12. A $\mathrm{H}_{2}$ image of the $\mathrm{L} 1340 \mathrm{C}$ region. Circles mark the positions of $\mathrm{H}_{2}$ shocks, and boxes indicate the locations of subsequent figures in the text.

\section{3. $L 1340 C$}

\subsubsection{The RNO9 Region}

The RNO9 region contains a grouping of stars roughly coincident with "a nebulous star that is bright at $2 \mu \mathrm{m}$ and invisible in the optical wavelengths" described by Kumar et al. (2002). In this grouping lies SSTSL2 J023127.34+724012.9, which Kun et al. (2016b) classified as a Class 0/I source. Just to the west of that source is a star visible in our $\mathrm{K}_{S}$ image that has a small bipolar reflection nebula oriented at about $\mathrm{PA} \sim 150^{\circ}$ around it (Figure 13). The star is invisible in our $\mathbf{J}$ image but bright at $\mathrm{H}$ and $\mathrm{K}_{S}$. The reflection nebula appears to have a disk shadow roughly perpendicular to the bipolar axis of the nebula in the $\mathrm{J}$ image, which disappears at $\mathrm{H}$ and $\mathrm{K}_{S}$. We find a $\mathrm{H}_{2}$ knot (MHO 2952) 39 arcsec southeast of this object at $\mathrm{PA} \sim 156^{\circ}$. Thus, we find it likely that the star with the small bipolar reflection nebula is driving an outflow along that axis.

\subsubsection{The L1340 C Mid-IR Cluster North: MHO 2953, MHO 2954, MHO 2959, MHO 2960}

Roughly 4.3 arcmin southeast of RNO9 is a grouping of three IRAS sources $(\mathrm{F} 02277+7226,02276+7225$, F02279
+7225) listed by Kun et al. (1994) as candidate young stars. The WISE $22 \mu \mathrm{m}$ image of this region reveals eight stars in a 2.7 diameter region. This cluster of infrared sources stands out from other regions of the L1340 cloud complex as being particularly dense. The only comparable cluster in the L1340 region is RNO7 in L1340 A.

There is a group of four $\mathrm{H}_{2}$ knots (MHO 2953, 2954, 2959, 2960) in the northern half of this cluster near IRAS F02277 +7226 . This is also roughly coincident with the $\mathrm{C}_{2} \mathrm{NH}_{3}$ core of Kun et al. (2003). Two Class 0/I sources from Kun et al. (2016b) lie in this region: SSTSL2 J023225.98+724020.1 and SSTSL2 J023237.90+723940.7.

MHO 2960 is coincident with SSTSL2 J023237.90 +723940.7 . The star is invisible in our $\mathrm{J}$ and $\mathrm{H}$ images, visible in our $\mathrm{K}_{S}$ image, but very bright in our $\mathrm{H}_{2}$ image (leading to our designation for it as an MHO object). MHO 2953 and MHO 2954 are faint, compact knots (Figure 14). MHO 2959 is a short (5 arcsec long) filament with a knot on the southwestern end that lies 54 arcsec to the northeast of the position of IRAS F02277+7226. Due to the numerous IRAS and WISE sources in the region, none of these four shocks can be positively associated with a source star in the region. 


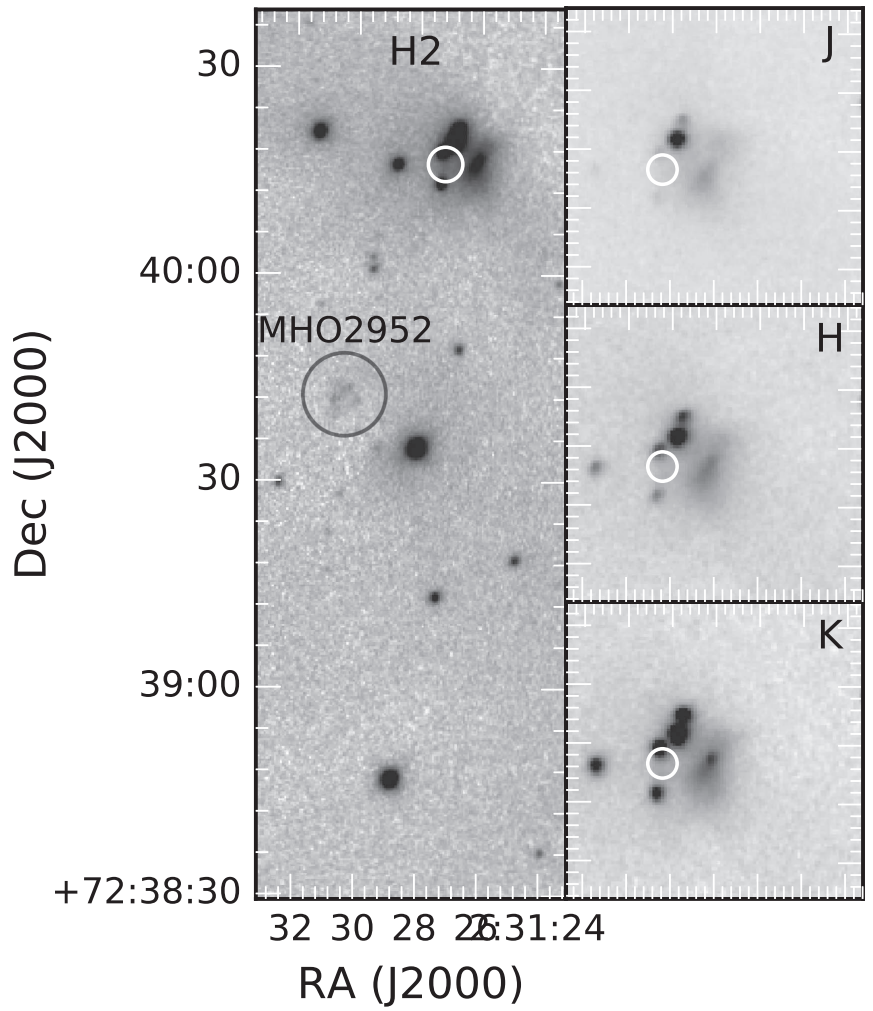

Figure 13. The left panel shows a $\mathrm{H}_{2}$ image of the MHO 2952 flow discussed in Section 3.3.1. The right-hand panels show broadband images of the source region with the reflection nebula discussed in the text. The white circle indicates the catalog position of the SSTSL source discussed in the text.

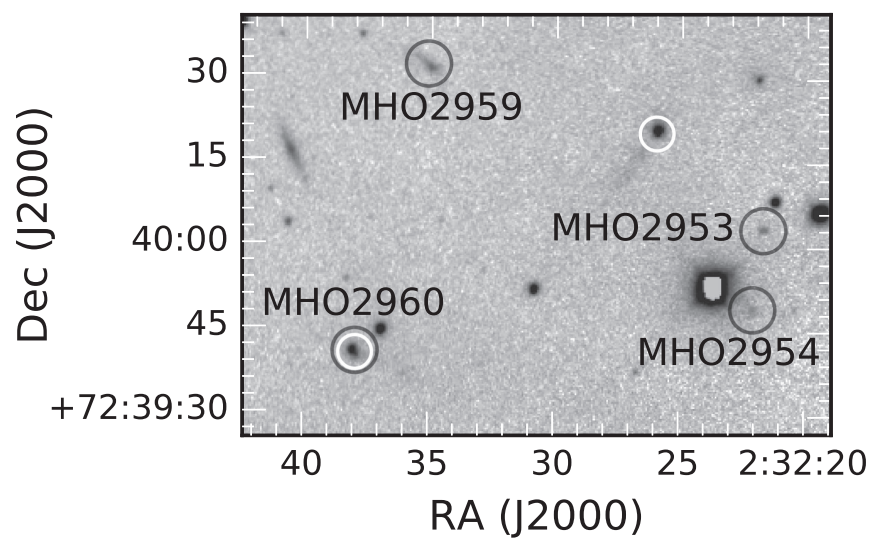

Figure 14. $\mathrm{A} \mathrm{H}_{2}$ image of the region discussed in Section 3.3 .2 containing MHO 2953, MHO 2954, MHO 2959, and MHO 2960. The white circles represent the positions of SSTSL2 J023225.98+724020.1 and SSTSL2 J023237.90+723940.7.

\subsubsection{The L1340 C Mid-IR Cluster South: MHO 2955, MHO 2956, MHO 2958, MHO 2963}

In the southern half of the cluster, roughly $45^{\prime \prime}$ south of IRAS F02279+7225 and IRAS $02276+7225$, we find a pair of stars separated by about 5 arcsec and surrounded by a reflection nebula (Figure 15). The southern star is coincident with the Kun et al. (2016b) Class 0/I source SSTSL2 J023232.00 +723827.5 . The reflection nebula is filamentary with one prominent filament offset from the stars to the southwest and oriented northwest-southeast. It is suggestive of the brightened limb of a cavity oriented in that direction because the region between the filament and the stars is noticeably darker (especially in $\mathrm{K}_{S}$ ). There is an additional bright knot of reflection nebulosity 19 arcsec northwest of the northern star. These stars are roughly coincident with the $\mathrm{C} 3 \mathrm{~W} \mathrm{NH} \mathrm{NH}_{3}$ core of Kun et al. (2003).

These stars are surrounded by three $\mathrm{H}_{2}$ knots (MHO 2955, 2956, and 2958). The brightest is MHO 2955, which lies about 20 arcsec west of the pair of stars. MHO 2956 is a faint, compact knot that lies about 9 arcsec northwest of the northern star. MHO 2958 lies 10 arcsec southeast of the southern star. In addition, MHO 2963 lies about $1^{\prime}$ to the east.

Lastly, about 1.85 arcmin to the west lies a fan-shaped reflection nebula with the Class 0/I source SSTSL2 J023207.96 +723759.3 at its apex. Both the star and reflection nebula are visible in the $\mathrm{K}_{S}$ and $\mathrm{H}_{2}$ images, but they are very faint in $\mathrm{J}$ and $\mathrm{H}$. No $\mathrm{H}_{2}$ knots are detected in the vicinity of this nebula.

\subsubsection{The V1180 Cas B Outflow: MHO 2964}

Roughly 5 arcmin to the north of the group of IRAS sources lies a beautiful S-shaped outflow (MHO 2964; Figure 16). The flow consists of eight distinct knots stretching over 1.75 arcmin along a north-south axis.

The flow axis passes near the position of two potential source stars, the brighter of which is the emission-line star V1180 Cas (emission-line star 72 in Table 2 of Kun et al. (2016a)). V1180 Cas coincides with SSTSL2 J023301.52 +724326.7, which Kun et al. (2016b) identified as a flat SED source. Antoniucci et al. (2014) examined the region around these two stars and designated the fainter star V1180 Cas B (which coincides with SSTSL2 J023302.41+724331.2, a class 0/I star cataloged by Kun et al. 2016b).

Both Antoniucci et al. (2014) and Kun et al. (2016b) detected MHO 2964 (in $\mathrm{H}_{2}$ and Spitzer $4.5 \mu \mathrm{m}$ bands, respectively). Our $\mathrm{H}_{2}$ images show that the MHO 2964 chain of knots clearly passes through V1180 Cas B (see Figure 16), so we favor this star as the driving source, as do Antoniucci et al. (2014) and Kun et al. (2016b). The star is visible in our H and $\mathrm{K}_{S}$ images, but it is invisible in our $\mathrm{J}$ image.

To the west of this source lie two additional $\mathrm{H}_{2}$ knots (MHO 2961 and MHO 2957; Figure 16). MHO 2961 is a 14 arcsec long filament oriented roughly northwest-southeast. MHO 2957 is a very faint, diffuse path of $\mathrm{H}_{2}$ emission. Neither has a clear association with a source.

\subsubsection{MHO 2962, MHO 2985, MHO 2966}

North of V1180 Cas lies a fan-shaped reflection nebula (Figure 17). At the apex of the nebula is a star visible in our J, $\mathrm{H}$, and $\mathrm{K}_{S}$ images that corresponds to the Class $0 / \mathrm{I}$ source SSTSL2 J023256.14+724605.3 of Kun et al. (2016b). To the northwest, 69 arcsec away along the axis of the fan $\left(\mathrm{PA} \sim 315^{\circ}\right.$ ) lies a compact $\mathrm{H}_{2}$ knot (MHO 2962). In addition, 6 arcmin to the southeast, along a similar axis $\left(\mathrm{PA} \sim 119^{\circ}\right)$, lies MHO 2966, which is a cluster of three faint bow shocks facing back along the flow axis.

The star at the apex of the reflection nebula is one of the outbursting stars identified by Kun et al. (2014). They also identified two $\mathrm{HH}$ objects in narrowband $\mathrm{H} \alpha$ and [S II]. The SE object (using their nomenclature) is coincident with our MHO 2962. There is no conclusive counterpart in our $\mathrm{H}_{2}$ images to their NW object. 


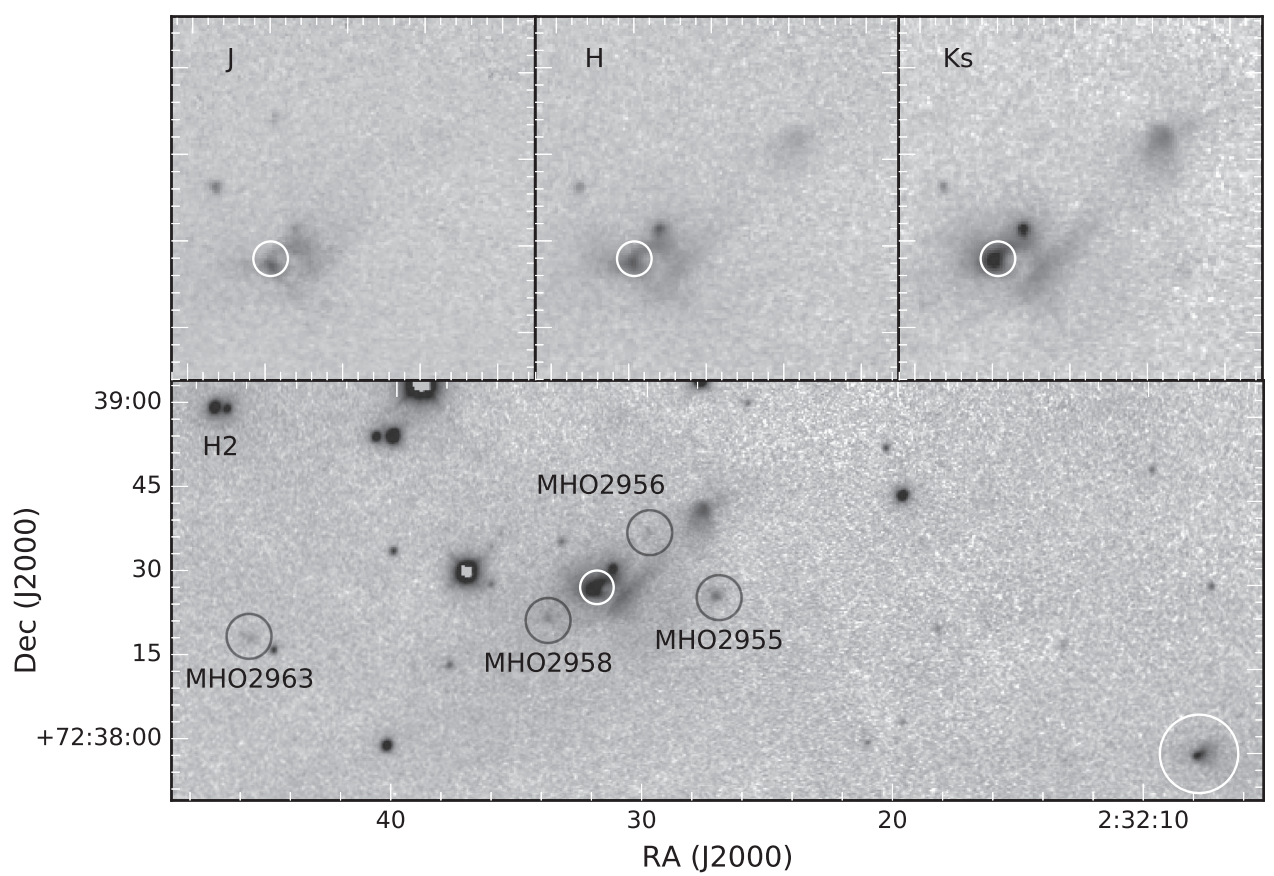

Figure 15. $\mathrm{A} \mathrm{H}_{2}$ image of the region discussed in Section 3.3.3 containing MHO 2955, MHO 2956, MHO 2958, and MHO 2963. The upper panels show broadband images of the reflection nebulosity near the center of the $\mathrm{H}_{2}$ image. The position of SSTSL2 J023232.00+723827.5 is marked with a white circle. The fan-shaped reflection nebula discussed in the text is indicated by a large white circle in the lower right corner of the $\mathrm{H}_{2}$ image.

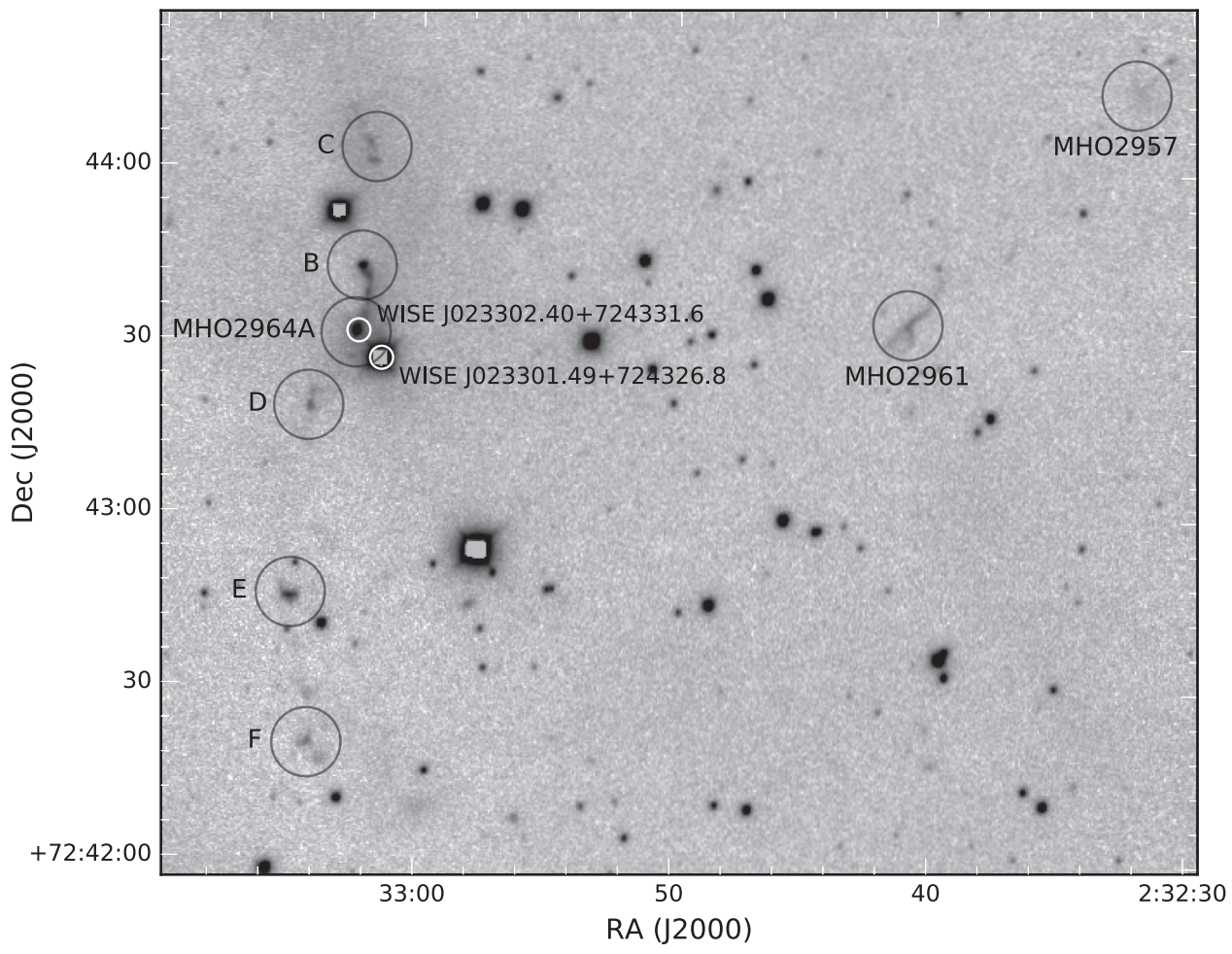

Figure 16. $\mathrm{A} \mathrm{H}_{2}$ image of the MHO 2964 flow discussed in Section 3.3.4. MHO 2957 and MHO 2961 are visible in the western half of the image. V1180 Cas and V1180 Cas B are marked with white circles and labeled.

A second Class 0/I source (STSL2 J023248.83+724635.4) lies on a line drawn between the reflection nebula around SSTSL2 J023256.1+724605.3 and MHO 2962. The star appears slightly nebulous in our $\mathrm{J}, \mathrm{H}$, and $\mathrm{K}_{S}$ images.

MHO 2965 (Figure 12) is a $\mathrm{H}_{2}$ knot that lies 2.6 arcmin north of the reflection nebula described in Section 3.3.5 above.

\section{DISCUSSION}

\subsection{Comparison with Barnard 1}

We have previously used a similar wide-field near-IR survey of shocks to examine the Barnard 1 region in Perseus (Walawender et al. 2009). At roughly $\sim 10^{3} M_{\odot}$, the L1340 complex is roughly comparable in gas mass to Barnard 1 , but 

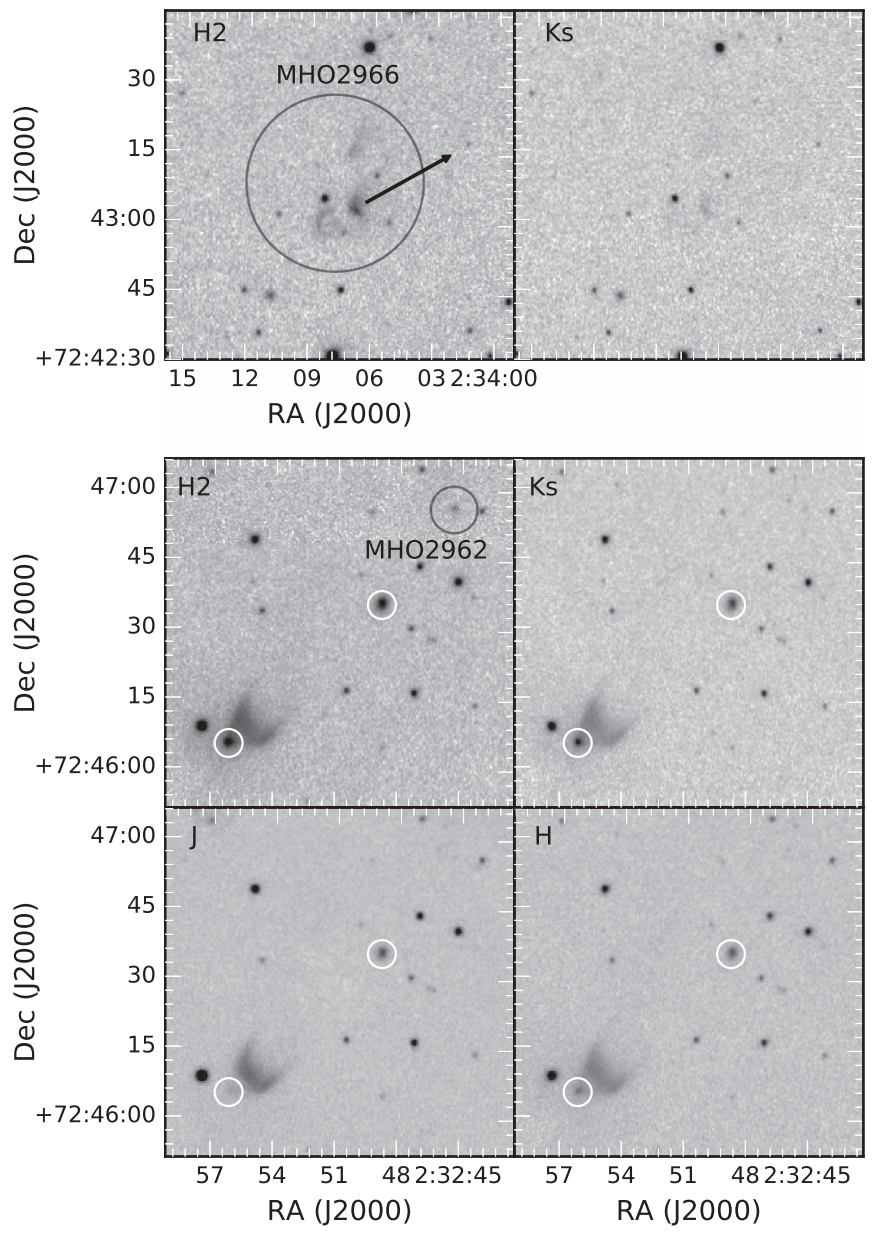

Figure 17. Images of MHO 2962 and MHO 2966 discussed in Section 3.3.5. The lower four panels show images of MHO 2962 and its associated reflection nebula. The positions of the two Class 0/I sources discussed in the text are marked with white circles. The upper two panels show the MHO 2966 shock, which is likely a shock in the counterflow to MHO 2962. The arrow indicates the direction to the reflection nebula and to SSTSL2 J023256.1+724605.3.

L1340 is divided up into three subcores (discussed in Sections 3.1-3.3 above), so it is morphologically different from the more cometary structure of Barnard 1. L1340 is also isolated, unlike Barnard 1, which is part of the larger Perseus molecular cloud complex, which contains several other regions of intense star-formation activity (Walawender et al. 2005). Barnard 1 also differs in that it is likely an example of triggered star formation. Kirk et al. (2006) used the offset between submillimeter clumps and their parent extinction cores within Perseus to show evidence that star formation in Perseus (including that in Barnard 1) has likely been triggered by soft ultraviolet radiation from a nearby B0.5 star, a hypothesis originally put forward by Walawender et al. (2004) based on the morphology of a single clump in the L1455 region of Perseus.

Outflow activity in L1340 appears to be somewhat more intense than in Barnard 1, with roughly twice the number of shock complexes (24 in Barnard 1 and 42 in L1340), but it is less concentrated in that outflows in Barnard 1 are dominated by those in a single central region with a few outliers, while outflows in L1340 are distributed among the three cores.

The very large, $3.7 \mathrm{pc}$ long outflow from SSTSL2 J022907.88+724347.2 (discussed in Section 3.1.1) is more than twice as long as the most extensive flow found in
Barnard 1 and appears to be only one-half of the flow as the northeastern lobe was not detected in our images. This gives it a dynamic age that is several times older than the oldest flow in Barnard 1 under similar assumptions about outflow launch velocity. This suggests that star formation in L1340 has been ongoing somewhat longer than in Barnard 1, but the SSTSL2 J022907.88+724347.2 outflow appears (in our J, H, and $\mathrm{K}_{S}$ images and by comparison with the Kun et al. (2016a) extinction map) to propagate through a comparatively low extinction regime. This could result in the flow not impacting as much ambient material, thus allowing it to travel at nearlaunch speeds over greater distances than flows that encounter surrounding material nearer their source protostar.

Despite the morphological differences (filamentary versus clumpy, triggered versus nontriggered), Barnard 1 and L1340 appear to have similar populations of outflows, with L1340's outflows likely being somewhat older.

\subsection{Properties of Outflow-driving Protostars}

In order to identify the candidate driving protostars for the outflows we described in Section 3, we considered both the morphology of the individual shocks (for example, the bow shock morphology of MHO 2925 discussed in Section 3.1.1 was used to estimate the direction in which the source protostar must lie) and the positions of the individual shocks relative to a candidate source (for example, see the discussion of the source region for MHO 2928 in Section 3.1.2). The candidate sources we considered consisted of $\mathrm{H} \alpha$ emission line stars identified in various previous works (primarily those in Kun et al. 2016a), IRAS sources identified as protostars in Kun et al. (1994), the 45 Class 0/I (eight of which they consider to be Class 0 candidates), and the 27 flat SED sources identified by Kun et al. (2016b).

Table 2 contains a list of the 12 shock complexes that we feel clearly represent a single outflow structure and for which we have identified at least one strong candidate source protostar. For only seven of those 12 outflows can we unambiguously identify a single source protostar; for the other five outflows, we are limited to identifying two to four candidate source protostars.

For the subset of these candidate outflow sources that are also classified as Class 0/I or flat SED, we can compare the properties derived by Kun et al. (2016b) of our candidate outflow source population with the properties of the Class $0 / \mathrm{I}$ or flat SED stars that are not outflow sources.

For each of the $A_{\mathrm{V}}, T_{\mathrm{bol}}$, and $L_{\mathrm{bol}}$ properties, we compute a Kolmogorov-Smirnov statistic to determine whether the values for source candidates are drawn from the same distribution as the non-outflow-source stars and determine a " $p$ value" that quantifies the chance that the source and nonsource protostar properties are drawn from the same distribution. Histograms for each property and the probability distribution function for obtaining each value are shown in Figure 18.

While all three properties have $p$ values which suggest that the two populations differ, none of the $p$ values are small enough to confidently reject the hypothesis that they are drawn from the same population.

Candidate outflow sources appear (at the $\sim 88 \%$ confidence level) to be drawn from a slightly lower $A_{\mathrm{V}}$ population than are the nonoutflow sources (see left column of Figure 18). This may be due, however, to selection effects. For example, shocks may be obscured in the highest-extinction regions, so shocks 
Table 2

Outflows in L1340

\begin{tabular}{lcllll}
\hline \hline Designation & Length $(', \mathrm{pc})$ & $\mathrm{NH}_{3}$ Core & Source Candidates & Class & Shock Components \\
\hline L1340A Flow 1 & $15 ! 5,3.7 \mathrm{pc}$ & $\mathrm{A} 3$ & SSTSL2 J022907.88+724347.2 & Flat SED & MHO 2925, 2937, HH 487 \\
L1340A Flow 2 & $5 ! 7,1.4 \mathrm{pc}$ & $\mathrm{A} 1$ & SSTSL2 J022818.51+723506.2 & Class 0/I & MHO 2928 \\
$\ldots$ & $\ldots$ & $\ldots$ & SSTSL2 J022820.81+723500.5 & Class 0 & $\ldots$ \\
L1340A Flow 3 & $9 ! 9,2.4 \mathrm{pc}$ & $\mathrm{A} 1$ & SSTSL2 J022844.40+723533.5 & Class 0/I & MHO 2926, 2935, 2936, 2938, 2940, HH 488 D, HH 672 \\
$\ldots$ & $\ldots$ & $\ldots$ & SSTSL2 J022842.57+723544.3 & Class 0/I & $\ldots$ \\
$\ldots$ & $\ldots$ & $\ldots$ & WISE J022817.97+723517.5 & $\ldots$ & $\ldots$ \\
L1340A Flow 4 & $4 ! 0,0.7 \mathrm{pc}$ & $\mathrm{A} 4$ & SSTSL2 J022943.01+724359.6 & Class 0/I & MHO 2939 \\
$\ldots$ & $\ldots$ & $\ldots$ & SSTSL2 J022943.64+724358.6 & Class 0/I & $\ldots$ \\
L1340B Flow 1 & $9 ! 1,2.2 \mathrm{pc}$ & $\ldots$ & SSTSL2 J022808.60+725904.5 & Class 0 & MHO 2942 \\
L1340B Flow 2 & $13 ! 3,3.2 \mathrm{pc}$ & $\ldots$ & SSTSL2 J022931.98+725912.4 & Class 0 & MHO 2941 \\
L1340B Flow 3 & $1 ! 0,0.24 \mathrm{pc}$ & $\ldots$ & SSTSL2 J022756.91+730354.4 & Class 0/I & MHO 2943 \\
L1340B Flow 4 & $4 ! 5,1.08 \mathrm{pc}$ & $\mathrm{B} 1$ & SSTSL2 J022955.10+730309.1 & Class 0/I & MHO 2946 \\
$\ldots$ & $\ldots$ & $\ldots$ & SSTSL2 J023020.61+730233.7 & Flat SED & $\ldots$ \\
L1340B Flow 5 & $0 ! 3,0.07 \mathrm{pc}$ & $\mathrm{B} 2$ & SSTSL2 J023042.36+730305.1 & Class 0/I & MHO 2947 \\
L1340C Flow 1 & $0 ! 65,0.16 \mathrm{pc}$ & $\ldots$ & WISE J023127.19+724015.9 & $\ldots$ & MHO 2952 \\
$\ldots$ & $\ldots$ & $\ldots$ & SSTSL2 J023127.34+724012.9 & Class 0/I & $\ldots$ \\
L1340C Flow 2 & $1 ! 75,0.30 \mathrm{pc}$ & $\ldots$ & SSTSL2 J023302.41+724331.2 & Class 0/I & MHO 2964 \\
L1340C Flow 3 & $1 ! 15,0.20 \mathrm{pc}$ & $\ldots$ & SSTSL2 J023256.14+724605.3 & Class 0 & MHO 2962 \\
\hline
\end{tabular}
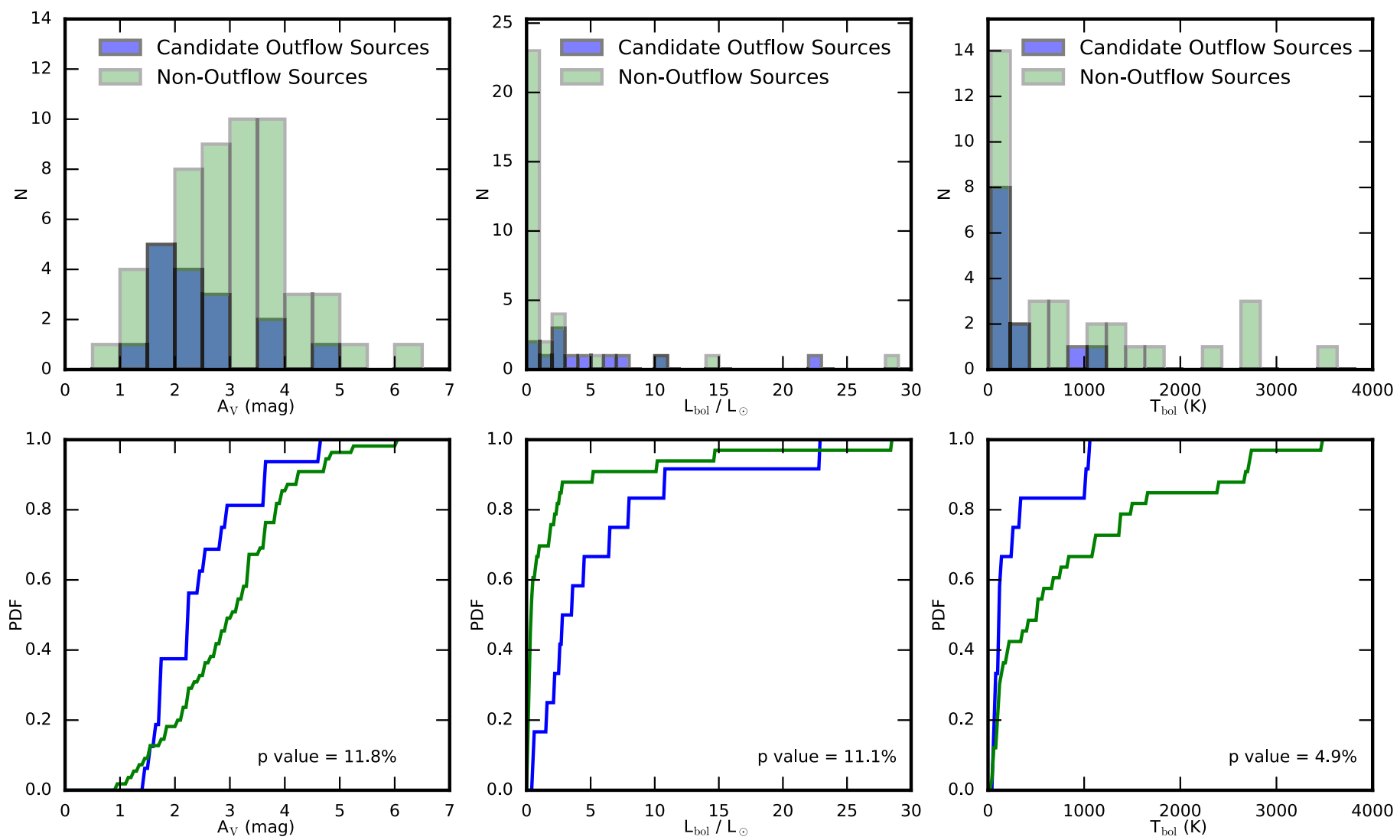

Figure 18. A comparison of the $A_{\mathrm{V}}, T_{\mathrm{bol}}$, and $L_{\mathrm{bol}}$ values (from Kun et al. (2016b) Tables 7 and 8) for stars that are candidate outflow sources compared with the entire population of Class $0 / \mathrm{I}$ and flat SED protostars. A histogram of the values for each population is shown in the top row, while the cumulative probability distribution function for each property is shown in the bottom row. The $p$ value quantifying the chances that these two populations are drawn from the same population is annotated in the lower right of each cumulative probability distribution function plot.

near the highest-extinction protostars may not be detected by our survey, and nearby shocks are the easiest to associate with a candidate protostar.

In the middle column of Figure 18 we see that the outflow source candidates lack the strong peak at low $L_{\mathrm{bol}}$ that the nonsource candidates do have. This is significant at a $\sim 89 \%$ confidence level. The candidate outflow sources are, on average, higher in luminosity than the nonsource candidates.

In the right-hand column of Figure 18, we see that both distributions are clustered around small values of $T_{\mathrm{bol}}$. However, from the cumulative probability distribution function in the right-hand panel, we see that proportionally more outflow source candidates are clustered at small values 


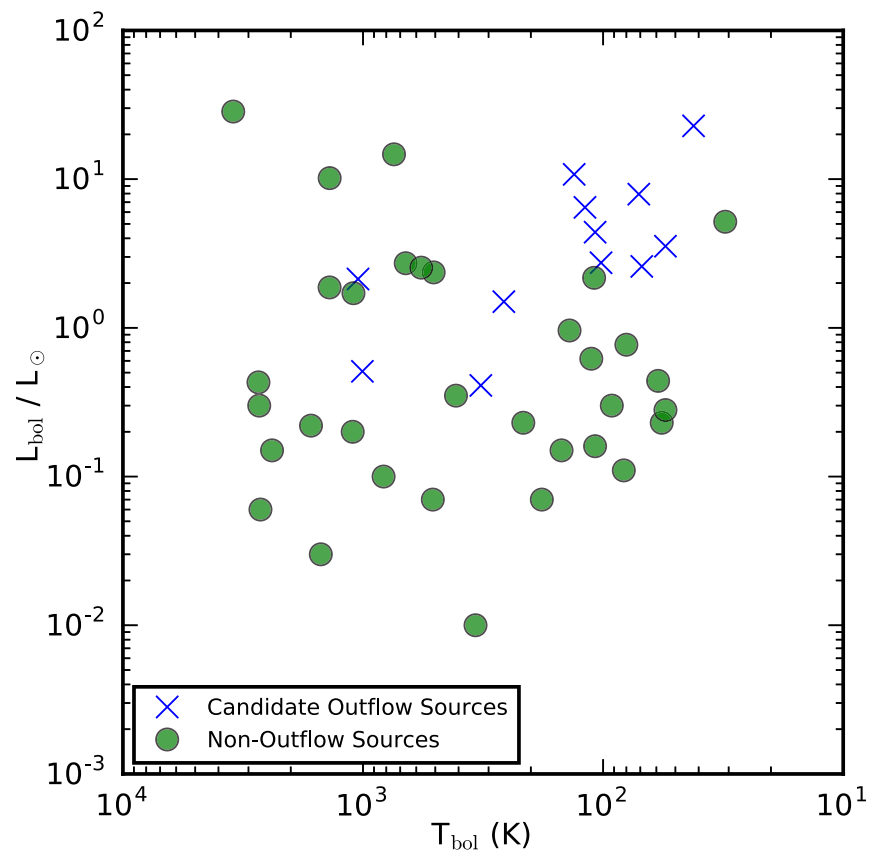

Figure 19. An $L_{\text {bol }}$ vs. $T_{\text {bol }}$ plot for the candidate outflow sources (marked with blue $\mathrm{x}$ symbols) and the nonoutflow sources (marked with solid green circles).

than are nonoutflow candidates. These two populations differ at the $\sim 95 \%$ confidence level. The bolometric temperature is a proxy for the evolutionary development of a protostar (Myers \& Ladd 1993). One would expect outflow-driving sources to be, on average, in the earlier stages of protostellar evolution and have lower bolometric temperatures, just as this result suggests.

We have also run the analysis above using only seven of the 12 outflow-driving protostars, selecting only those seven for which there was an unambiguous identification of the source. The statistical significance of this analysis with only seven sources is limited, but the qualitative results are similar: the $p$ value for $A_{\mathrm{V}}$ increases to $\sim 33 \%$ (making a selection effect less significant), while the $p$ values for $T_{\mathrm{bol}}$ and $L_{\mathrm{bol}}$ decrease to $\sim 1 \%$ and $\sim 7 \%$, respectively (nominally making the effect more significant).

We can also examine where outflow source candidates lie in a $L_{\text {bol }}$ versus $T_{\text {bol }}$ plot in comparison to the nonoutflow candidates. This is analogous to Figure 12 in Kun et al. (2016b), but only including Class 0/I and flat SED sources. This is shown in Figure 19, where the outflow source candidates can be seen to be biased toward the low $T_{\text {bol }}$ and high $L_{\mathrm{bol}}$ side of the overall population of sources, as would be expected based on the behaviors seen in Figure 18 above.

We also examined the source properties as a function of outflow length, but no correlation with any of $A_{\mathrm{V}}, T_{\mathrm{bol}}$, or $L_{\mathrm{bol}}$ was apparent.

\section{SUMMARY}

We have found 42 distinct shock complexes (MHO 2925-2966) in the L1340 region. Of those 42 shock complexes, we were able to link 17 of them into 12 distinct outflows with candidate source stars (Table 2). Of those 12 flows, four lie in L1340 A, five in L1340 B, and three in L1340 C. Six flows are longer than $1 \mathrm{pc}$ in length at the assumed distance to L1340 of $825 \mathrm{pc}$.
The shocks that we were not able to link into coherent flows tend to be concentrated in groups near the centers of the three regions. In L1340 A, five unassociated shocks lie within 1!7 of the RNO7 cluster (Section 3.1.5). In L1340 B, three unassociated shocks lie within 1.5 of RNO8 (Section 3.2.4). Lastly, in L1340 C, eight unassociated shocks lie within a 1.5 radius of a group of IRAS sources (Sections 3.3.2 and 3.3.3). Thus, 34 of our 42 shocks lie in identifiable outflows or in the more active (and thus confused) centers of the cloud. Only eight unassociated shocks lie in the outer areas of the cloud.

By combining our identification of candidate outflowproducing protostars with the Kun et al. (2016b) catalog of Class 0/I and flat SED protostars in this region, we can begin to compare the properties of protostars that drive outflows with those protostars that do not appear to drive outflows. With the work presented here, we have only a small sample, which may suffer from selection effects, but we see some hints (at a level less than $2 \sigma$ confidence) that the properties $\left(A_{\mathrm{V}}, T_{\mathrm{bol}}\right.$, and $\left.L_{\mathrm{bol}}\right)$ of outflow-driving protostars differ from non-outflow-driving protostars. Outflow-driving protostars appear to have slightly lower extinction (which could be a result of selection effects), have higher bolometric luminosity, and have lower bolometric temperatures (which, if we assume younger protostars are more likely to drive outflows, is consistent with the Myers \& Ladd (1993) use of bolometric temperature as a proxy for a protostar's evolutionary state).

This work is based on observations obtained with WIRCam, a joint project of CFHT, Taiwan, Korea, Canada, and France, at the Canada-France-Hawaii Telescope (CFHT) which is operated by the National Research Council (NRC) of Canada, the Institute National des Sciences de l'Univers of the Centre National de la Recherche Scientifique of France, and the University of Hawaii.

This work is based on observations obtained with the Apache Point Observatory $3.5 \mathrm{~m}$ telescope, which is owned and operated by the Astrophysical Research Consortium.

This publication makes use of data products from the Widefield Infrared Survey Explorer, which is a joint project of the University of California, Los Angeles, and the Jet Propulsion Laboratory/California Institute of Technology, funded by the National Aeronautics and Space Administration.

This research has made use of the VizieR catalog access tool, CDS, Strasbourg, France.

The MHO catalog is hosted by Liverpool John Moores University.

This research made use of Astropy, a community-developed core Python package for astronomy (Collaboration et al. 2013).

GW-C gratefully acknowledges support from the Brinson Foundation in aid of astrophysics research at the Adler Planetarium.

We would like to thank Adam Draginda, Rachael Zelman, and Mary Laychak (the CFHT queue observers), as well as Pierre Martin, Daniel Devost, and Todd Burdullis (the CFHT queue coordinators), who obtained our WIRCam data.

Finally, we would like to thank the University of Hawaii Time Allocation Committee for allocating the nights during which these observations were made.

The authors also wish to recognize and acknowledge the very significant cultural role and reverence that the summit of Mauna Kea has always had within the indigenous Hawaiian 
community. We are fortunate to have the opportunity to conduct observations from this sacred mountain.

Facilities: CFHT (WIRCAM), ARC (NICFPS), WISE.

\section{REFERENCES}

Antoniucci, S., Arkharov, A. A., Di Paola, A., et al. 2014, A\&A, 565, L7

Arce, H. G., \& Goodman, A. A. 2002, ApJ, 575, 911

Bally, J., Reipurth, B., Lada, C. J., \& Billawala, Y. 1999, AJ, 117, 410

Cohen, M. 1980, AJ, 85, 29

Collaboration, T. A., Robitaille, T. P., Tollerud, E. J., et al. 2013, A\&A, 558,33

Davis, C. J., Gell, R., Khanzadyan, T., Smith, M. D., \& Jenness, T. 2010, A\&A, 511, 24

Dorschner, J., \& Gürtler, J. 1963, AN, 287, 257

Hearty, F. R., Morse, J., Beland, S., et al. 2004, Proc. SPIE, 5492, 1623

Juvela, M., Ristorcelli, I., Pagani, L., et al. 2012, A\&A, 541, 12

Kirk, H., Johnstone, D., \& Di Francesco, J. 2006, ApJ, 646, 1009

Koenig, X. P., \& Leisawitz, D. T. 2014, ApJ, 791, 131
Kumar, M. S. N., Anandarao, B. G., \& Yu, K. C. 2002, AJ, 123, 2583

Kun, M., Apai, D., O'Linger-Luscusk, J., et al. 2014, ApJL, 795, L26

Kun, M., Moór, A., Szegedi-Elek, E., \& Reipurth, B. 2016a, ApJ, 822, 79

Kun, M., Obayashi, A., Sato, F., et al. 1994, A\&A, 292, 249

Kun, M., Szegedi-Elek, E., Moór, A., et al. 2011, ApJL, 733, L8

Kun, M., Wolf-Chase, G., Moór, A., et al. 2016b, ApJS, 224, 22

Kun, M., Wouterloot, J. G. A., \& Tóth, L. V. 2003, A\&A, 398, 169

Lynds, B. T. 1962, ApJS, 7, 1

Magakian, T. Y., Movsessian, T. A., \& Nikogossian, E. G. 2003, Ap, 46, 1 (English translation of Astrofizika)

Miesch, M. S., \& Bally, J. 1994, ApJ, 429, 645

Myers, P. C., \& Ladd, E. F. 1993, ApJL, 413, L47

Puget, P., Stadler, E., Doyon, R., et al. 2004, Proc. SPIE, 5492, 978

Quillen, A. C., Thorndike, S. L., Cunningham, A., et al. 2005, ApJ, 632, 941

Walawender, J., Bally, J., \& Reipurth, B. 2005, AJ, 129, 2308

Walawender, J., Bally, J., Reipurth, B., \& Aspin, C. 2004, AJ, 127, 2809

Walawender, J., Reipurth, B., \& Bally, J. 2009, AJ, 137, 3254

Yonekura, Y., Dobashi, K., Mizuno, A., Ogawa, H., \& Fukui, Y. 1997, ApJS, 110,21 